This is an Accepted Manuscript of an article published by Taylor \& Francis in "International Journal of Systems Science" on $06^{\text {th }}$ April 2016, available online:

http://wwww.tandfonline.com/10.1080/00207721.2016.1165898 


\title{
Robust unknown input observer for state and fault estimation in discrete-time Takagi-Sugeno systems
}

\author{
Damiano Rotondo ${ }^{a, *}$, Marcin Witczak $^{b}$, Vicenç Puig $^{a, c}$, Fatiha Nejjari $^{a}$, Marcin Pazera $^{b}$ \\ ${ }^{a}$ Automatic Control Department, Universitat Politècnica de Catalunya (UPC), Rambla de Sant Nebridi 11, \\ 08222 Terrassa, Spain. \\ ${ }^{b}$ Institute of Control and Computation Engineering, University of Zielona Gora, ul. Podgórna 50, 65-246 Zielona \\ Góra, Poland.
}

${ }^{c}$ Institut de Robòtica i Informàtica Industrial (IRI), UPC-CSIC, Carrer de Llorens i Artigas 4-6, 08028

Barcelona, Spain.

(Received 00 Month 20XX; final version received 00 Month 20XX)

\begin{abstract}
In this paper, a robust unknown input observer (UIO) for the joint state and fault estimation in discrete-time Takagi-Sugeno (TS) systems is presented. The proposed robust UIO, by applying the $\mathcal{H}_{\infty}$ framework, leads to a less restrictive design procedure with respect to recent results found in the literature. The resulting design procedure aims at achieving a prescribed attenuation level with respect to the exogenous disturbances, while obtaining at the same time the convergence of the observer with a desired bound on the decay rate. An extension to the case of unmeasurable premise variables is also provided. Since the design conditions reduce to a set of linear matrix inequalities (LMIs), that can be solved efficiently using the available software, an evident advantage of the proposed approach is its simplicity. The final part of the paper presents an academic example and a real application to a multi-tank system, which exhibit clearly the performance and effectiveness of the proposed strategy.
\end{abstract}

Keywords: State estimation, fault diagnosis, unknown input observers (UIO), Takagi-Sugeno (TS) fuzzy systems.

\section{Introduction}

Fault detection and isolation (FDI) systems have been a very active area of research in the last decades and, consequently, many schemes for FDI have been developed (see Zhang and Jiang (2008); Hwang et al. (2010); Samy et al. (2011)). The FDI approaches, such as neural-network-based methods (Patan et al. 2008) and identification-based methods (Simani et al. 2003), are generally classified into modelbased/data-based and quantitative/qualitative techniques (Zhang and Jiang 2008). A quantitative modelbased FDI scheme utilizes a mathematical model, often known as analytical redundancy, to carry out FDI in real-time.

Among the proposed solutions for fault diagnosis systems, the observer-based ones have gained a lot of interest. These fault estimation methods attempt to reconstruct the fault rather than to detect its presence, and provide a direct estimate of its magnitude and severity, which is important in many applications, especially when an active fault-tolerant control (FTC) strategy is implemented (Mahmoud et al. 2003; Noura et al. 2009; Witczak 2014). Among these techniques, there are Kalman filter-based schemes (Keller and Darouach 1999), minimum-variance estimators (Gillijns and Moor 2007), adaptive estimators (Zhang et al. 2010), sliding mode observers (Xu et al. 2012; Brahim et al. 2015) and adaptive observers (Rotondo et al. 2014).

\footnotetext{
* Corresponding author. e-mail: damiano.rotondo@yahoo.it
} 
Takagi-Sugeno (TS) systems, as introduced by Takagi and Sugeno (1985), provide an effective way of representing nonlinear systems with the aid of fuzzy sets, fuzzy rules and a set of local linear models which are smoothly connected by fuzzy membership functions (Feng 2006). TS fuzzy models are universal approximators since they can approximate any smooth nonlinear function to any degree of accuracy (Johansen et al. 2000), such that they can represent complex nonlinear systems. Different observer design techniques have been developed in the literature for TS systems (Ichalal et al. 2010, 2009; Chadli et al. 2009; Bouattour et al. 2010; Moodi and Farrokhi 2013; K. Zhang and Shi 2009).

The state observation for dynamic systems with unknown inputs or disturbances has become of paramount importance both from the theoretical and the practical points of view. For this reason, starting from the seminal work by Wang et al. (1975), the research interest has been attracted by the problem of designing unknown input observers (UIOs), and a lot of effort has been put into developing this technique in the last decades (see Witczak (2007), Witczak (2014), and the references therein). The capacity of estimating the state in the presence of unknown inputs has particular relevance in the design of FDI schemes, as suggested in recent works (Chen and Saif 2007, 2010; Jia et al. 2011; Fonod et al. 2014). In particular, the design of UIOs for TS systems has been an interesting topic of research in recent years (Chadli 2010; Chadli and Karimi 2013).

In this paper, a robust UIO for the joint state and fault estimation in discrete-time TS systems is proposed. The resulting design procedure aims at achieving a prescribed attenuation level with respect to the exogenous disturbances, while obtaining at the same time the convergence of the observer with a desired bound on the decay rate. The problem is addressed in both the cases of measurable and unmeasurable premise variables. One advantage of the proposed approach is its simplicity in reducing the design conditions to a set of linear matrix inequalities (LMIs), that can be solved efficiently using the available software. An academic example and a real application to a multi-tank system show clearly the effectiveness of the proposed strategy.

This paper is structured as follows. Section 2 formulates the problem, and revisits the recent result developed in Chadli and Karimi (2013), in order to show the limitations that are overcome by the proposed approach. Section 3 presents the main results of the paper, i.e. the definition of the UIO, the design procedure with and without convergence rate specifications, and the fault estimation. In Section 4, two illustrative examples are used to show the effectiveness of the technique. Finally, the main conclusions are drawn in Section 5.

\section{Preliminaries and problem formulation}

Consider the following TS fuzzy model:

$$
\begin{gathered}
x_{k+1}=A\left(s_{k}\right) x_{k}+B\left(s_{k}\right) u_{k}+B\left(s_{k}\right) f_{k}+W_{1}\left(s_{k}\right) w_{k} \\
=\sum_{i=1}^{M} h_{i}\left(s_{k}\right)\left[A^{i} x_{k}+B^{i} u_{k}+B^{i} f_{k}+W_{1}^{i} w_{k}\right] \\
y_{k}=C\left(s_{k}\right) x_{k}+W_{2}\left(s_{k}\right) w_{k} \\
=\sum_{i=1}^{M} h_{i}\left(s_{k}\right)\left[C^{i} x_{k}+W_{2}^{i} w_{k}\right]
\end{gathered}
$$

with:

$$
h_{i}\left(s_{k}\right) \geq 0 \quad \forall i=1, \ldots, M \quad \sum_{i=1}^{M} h_{i}\left(s_{k}\right)=1
$$


where $x_{k} \in \mathrm{R}^{n}$ stands for the state, $y_{k} \in \mathrm{R}^{m}$ is the output, $u_{k} \in \mathrm{R}^{r}$ denotes the nominal control input, $f_{k} \in \mathrm{R}^{r}$ is the actuator fault, and $w_{k} \in l_{2}$ is a an exogenous disturbance vector satisfying:

$$
\begin{aligned}
& l_{2}=\left\{w \in \mathrm{R}^{n} \mid\|w\|_{l_{2}}<+\infty\right\} \\
& \|w\|_{l_{2}}=\left(\sum_{k=0}^{\infty}\left\|w_{k}\right\|^{2}\right)^{\frac{1}{2}}
\end{aligned}
$$

The activation functions $h_{i}(\cdot)$ depend on the vector of premise variables $s_{k}=\left[s_{k}^{1}, s_{k}^{2}, \ldots, s_{k}^{p}\right]^{T}$, which is assumed to depend on measurable variables, e.g. system outputs and known inputs (Takagi and Sugeno 1985) (however, this assumption will be later relaxed by considering the case of unmeasurable premise variables).

Notice that (2) describes systems with a time-varying output equation and thus is a more general representation than the one with constant matrices $C$ and $W$, which constitutes a special case of (2). For R3-1

instance, cases for which this generalization could be of interest comprehend systems with nonlinear sensors (e.g. Cotton and Wilamowski (2010)) or state-space models identified using black-box identification (Vizer et al. 2013).

It is desired to achieve the following goals:

- to obtain an estimation of the states using an UIO, taking into account the actuator fault $f_{k}$ as an unknown input;

- to estimate the actuator fault $f_{k}$ using the state estimation provided by the UIO.

Hereafter, a short review of the recent result (Chadli and Karimi 2013) is performed, in order to compare it with the approach presented in the remaining of the paper. The TS fuzzy models considered in Chadli and Karimi (2013) are represented by:

$$
\begin{aligned}
x_{k+1} & =\sum_{i=1}^{M} h_{i}\left(s_{k}\right)\left[A^{i} x_{k}+B^{i} u_{k}+B^{i} f_{k}+W_{1}^{i} w_{k}\right] \\
y_{k} & =C x_{k}+F f_{k}+W_{2} w_{k}
\end{aligned}
$$

while the associated UIO is:

$$
\begin{aligned}
z_{k+1} & =\sum_{i=1}^{M} h_{i}\left(s_{k}\right)\left[N^{i} z_{k}+G^{i} u_{k}+L^{i} y_{k}\right] \\
\hat{x}_{k} & =z_{k}-E y_{k}
\end{aligned}
$$

Let us define the state estimation error $e_{k}=x_{k}-\hat{x}_{k}$, which taking into account (6)-(9) gives:

$$
\begin{aligned}
e_{k+1}= & \sum_{i=1}^{M} h_{i}\left(s_{k}\right)\left[N^{i} e_{k}+\left(T A^{i}-K^{i} C-N^{i}\right) x_{k}+\left(T B^{i}-G^{i}\right) u_{k}\right. \\
& \left.+\left(T B^{i}-K^{i} F\right) f_{k}+\left(T W_{1}^{i}-K^{i} W_{2}\right) w_{k}+E F f_{k+1}+E W_{2} w_{k+1}\right]
\end{aligned}
$$

with:

$$
T=I-E C, \quad K^{i}=N^{i} E+L^{i}
$$


which under:

$$
\begin{array}{r}
N^{i}=T A^{i}-K^{i} C \\
T B^{i}-G^{i}=0 \\
T B^{i}-K^{i} F=0 \\
E\left[F \quad W_{2}\right]=0 \\
T W_{1}^{i}-K^{i} W_{2}=0
\end{array}
$$

boils down to:

$$
e_{k+1}=\sum_{i=1}^{M} h_{i}\left(s_{k}\right) N^{i} e_{k}
$$

Subsequently, Chadli and Karimi (2013) show that the design procedure, which guarantees that $e_{k}$ converges asymptotically to zero, can be reduced to solving a relatively simple set of LMIs.

The approach proposed by Chadli and Karimi (2013) has an incontestable appeal, also due to the fact that it considers an unknown input in the output equation, which may represent a sensor fault. However, it has the following limitations:

- the matrices $C$ and $W_{2}$ in the output equation (7) are constant, whereas the matrices in (2) are time-varying combinations of $C^{i}$ and $W_{2}^{i}$;

- the external disturbance $w_{k}$ is eliminated from (10), which requires that (15)-(16) hold. This can be realized under perfect knowledge about $W_{1}$ and $W_{2}$, which is rather unrealistic to have in practice;

- a single matrix $T$ has to satisfy (14) for $i=1, \ldots, M$;

- no solution for estimating $f_{k}$ is provided in Chadli and Karimi (2013).

In the following section, a novel approach that overcomes these limitations will be proposed.

\section{Main results}

\subsection{Full rank condition}

Following Gillijns and Moor (2007) and Witczak (2007, 2014), let us assume that for (1)-(2) the rank condition:

$$
\operatorname{rank}\left(C\left(s_{k+1}\right) B\left(s_{k}\right)\right)=\operatorname{rank}\left(B\left(s_{k}\right)\right)=r \quad \forall s_{k}
$$

R3-2 is satisfied. As demonstrated in the subsequent part of the paper, under the above rank condition it is possible to derive an exact algebraic formulae, which uniquely describes the fault. This guarantees uniqueness and identifiability of the fault. If this condition were not satisfied, one could use an adaptive approach (see, e.g., (Witczak et al. 2015) and the references therein) or decompose the original term $B\left(s_{k}\right)$ into:

$$
B\left(s_{k}\right)=B_{1}\left(s_{k}\right) B_{2}\left(s_{k}\right)
$$

with $B_{1}\left(s_{k}\right)$ having the desired rank property.

Notice that the rank condition (18) is equivalent to:

$$
\operatorname{rank}\left(\sum_{j=1}^{M} h_{j}\left(s_{k+1}\right) \sum_{i=1}^{M} h_{i}\left(s_{k}\right) C^{j} B^{i}\right)=\operatorname{rank}\left(\sum_{i=1}^{M} h_{i}\left(s_{k+1}\right) B^{i}\right)=r
$$


Then, the problem boils down to checking the full rank property of all convex combinations of $B^{i}, i=$ $1, \ldots, M$ as well as $C^{j} B^{i}, i=1, \ldots, M, j=1, \ldots, M$.

Let us consider the problem of checking the full rank property of all convex combinations of $B^{i}, i=$ $1, \ldots, M$. Notice that the task of checking the full rank property of $C^{j} B^{i}, i=1, \ldots, M, j=1, \ldots, M$ can be done in the same way.

First of all, let us recall that a matrix $\Xi \in \mathrm{R}^{n \times n}$ is called a $P$-matrix if all its principal minors are positive (Elsner et al. 2002). On the other hand, a matrix $\Xi \in \mathrm{R}^{n \times n}$ is a block P-matrix with respect to a partition $N(\lambda)$ of $N=\{1, \ldots, n\}$ into $\lambda \in[1, n]$ pairwise disjoint nonvoid subsets $N_{i}$ of cardinality $n_{i}$, $i=1, \ldots, \lambda$, if for any $T \in \mathcal{T}_{n}^{\lambda}$ (see (Witczak et al. 2015) for a detailed explanation):

$$
\operatorname{det}(T \Xi+(I-T)) \neq 0
$$

where $\mathcal{T}_{n}^{\lambda}$ is the set of all diagonal matrices $T \in \mathrm{R}^{n \times n}$ such that $T\left[N_{i}\right]=t_{i} I, t_{i} \in[0,1], i=1, \ldots, \lambda$, where $T\left[N_{i}\right]$ is the principal submatrix of $T$ with row and column indices in $N_{i}$ (Elsner et al. 2002). A P-matrix is also block P-matrix with respect to any partition (Elsner et al. 2002). P-matrices and block P-matrices play an important property in studying the nonsingularity, Schur and Hurwitz stability of convex combinations of matrices (Johnson and Tsatsomeros 1995; Elsner and Szulc 1998, 2002).

Let us assume that the matrices $B^{i}, i=1, \ldots, M$, are full rank (if not, it can be already concluded that the full rank property does not hold), and let us define:

$$
\begin{aligned}
& Q_{p, p}=B^{p T} B^{p}, \quad p=1, \ldots, M \\
& Q_{p, a}=B^{p T} B^{a}+B^{a T} B^{p}-B^{a T} B^{a}-B^{p T} B^{p} \quad \text { for } p<a
\end{aligned}
$$

$$
R_{a, b}^{p}= \begin{cases}Q_{p, p} & \text { if }(a, b)=(1,1) \\ Q_{b-1, p} & \text { if } a=1 \wedge b=2, \ldots, p \\ I & \text { if } a=b \wedge 1<b<k \\ -I & \text { if } b=1 \wedge a=p+1 \\ 0 & \text { otherwise }\end{cases}
$$

Theorem 1. (Kolodziejczak and Szulc 1999) The following are equivalent:

(a) All convex combinations of $B^{1}, \ldots, B^{M}$ have full rank.

(b) $B^{M}$ has full row rank and the $(M-1) M n$-by- $(M-1) M n$ matrix:

$$
V=\left[\begin{array}{ccccc}
R_{1} R_{M}^{-1} & V_{1,2} & V_{1,3} & \ldots & V_{1,4} \\
-I_{M n} & I_{M n} & 0_{M n} & \ldots & 0_{M n} \\
0_{M n} & -I_{M n} & I_{M n} & \ldots & 0_{M n} \\
\ldots & \ldots & \ldots & \ldots & \ldots \\
0_{M n} & \ldots & 0_{M n} & -I_{M n} & I_{M n}
\end{array}\right]
$$

where $V_{1,2}=\left(R_{2}-R_{1}\right) R_{M}^{-1}, V_{1,3}=\left(R_{3}-R_{2}\right) R_{M}^{-1}$ and $V_{1,4}=\left(R_{M-1}-R_{M-2}\right) R_{M}^{-1}$ is a block $P$ matrix (Kolodziejczak and Szulc 1999) with respect to the partition $\left\{F_{1}, \ldots, F_{M-1}\right\}$ of $\{1, \ldots,(M-$ 1) $M n\}$, with $F_{i}=\{(M-1) M n+1, \ldots, i M n\}, i=1, \ldots, M-1$.

Proof. See Kolodziejczak and Szulc. (1999).

Having a tool for checking condition (20), it is possible to derive the observer design procedure, which is the main result of this paper.

\subsection{Unknown input observer}

By combining (1) and (2), the following is obtained:

$$
C\left(s_{k+1}\right) B\left(s_{k}\right) f_{k}=y_{k+1}-C\left(s_{k+1}\right) A\left(s_{k}\right) x_{k}-C\left(s_{k+1}\right) B\left(s_{k}\right) u_{k}-C\left(s_{k+1}\right) W_{1}\left(s_{k}\right) w_{k}-W_{2}\left(s_{k+1}\right) w_{k+1}
$$


Notice that (26) is an identity, since for given matrices $A\left(s_{k}\right), B\left(s_{k}\right), C\left(s_{k+1}\right)$, and vectors $f_{k}, x_{k}, u_{k}$, $w_{k}, w_{k+1}$, the value of the vector $y_{k+1}$ cannot be arbitrary, but is determined by (1)-(2). It follows that if $f_{k}$ was considered an unknown variable, the linear system of equations resulting from (26) would admit a solution (i.e. the actual value of $f_{k}$ ), that could be obtained as:

$$
\begin{aligned}
f_{k}= & H\left(s_{k}, s_{k+1}\right) y_{k+1}-H\left(s_{k}, s_{k+1}\right) C\left(s_{k+1}\right) A\left(s_{k}\right) x_{k}-u_{k} \\
& -H\left(s_{k}, s_{k+1}\right) C\left(s_{k+1}\right) W_{1}\left(s_{k}\right) w_{k}-H\left(s_{k}, s_{k+1}\right) W_{2}\left(s_{k+1}\right) w_{k+1}
\end{aligned}
$$

where $H\left(s_{k}, s_{k+1}\right)$ denotes the Moore-Penrose pseudoinverse of $C\left(s_{k+1}\right) B\left(s_{k}\right)$.

Due to the rank condition (18), (27) is the unique solution of the linear system obtained from (26). Moreover, $H\left(s_{k}, s_{k+1}\right)$ can be calculated easily as:

$$
H\left(s_{k}, s_{k+1}\right)=\left(C\left(s_{k+1}\right) B\left(s_{k}\right)\right)^{\dagger}=\left[\left(C\left(s_{k+1}\right) B\left(s_{k}\right)\right)^{T} C\left(s_{k+1}\right) B\left(s_{k}\right)\right]^{-1}\left(C\left(s_{k+1}\right) B\left(s_{k}\right)\right)^{T}
$$

where $\dagger$ denotes the Moore-Penrose pseudoinverse.

Introducing (27) into (1) leads to:

$$
x_{k+1}=\bar{A}\left(s_{k}, s_{k+1}\right) x_{k}+\bar{H}\left(s_{k}, s_{k+1}\right) y_{k+1}+\bar{W}_{1}\left(s_{k}, s_{k+1}\right) w_{k}+\bar{W}_{2}\left(s_{k}, s_{k+1}\right) w_{k+1}
$$

or, alternatively:

$$
x_{k}=\bar{A}\left(s_{k-1}, s_{k}\right) x_{k-1}+\bar{H}\left(s_{k-1}, s_{k}\right) y_{k}+\bar{W}_{1}\left(s_{k-1}, s_{k}\right) w_{k-1}+\bar{W}_{2}\left(s_{k-1}, s_{k}\right) w_{k}
$$

with:

$$
\begin{gathered}
\bar{A}\left(s_{k-1}, s_{k}\right)=\left(I-B\left(s_{k-1}\right) H\left(s_{k-1}, s_{k}\right) C\left(s_{k}\right)\right) A\left(s_{k-1}\right) \\
\bar{H}\left(s_{k-1}, s_{k}\right)=B\left(s_{k-1}\right) H\left(s_{k-1}, s_{k}\right) \\
\bar{W}_{1}\left(s_{k-1}, s_{k}\right)=\left(I-B\left(s_{k-1}\right) H\left(s_{k-1}, s_{k}\right) C\left(s_{k}\right)\right) W_{1}\left(s_{k-1}\right) \\
\bar{W}_{2}\left(s_{k-1}, s_{k}\right)=B\left(s_{k-1}\right) H\left(s_{k-1}, s_{k}\right) W_{2}\left(s_{k}\right)
\end{gathered}
$$

Then, using some technique, e.g. the well-known sector nonlinearity approach (Tanaka and Wang 2001; Rotondo et al. 2015), it is possible to obtain a TS model for (29) and (2), as follows ${ }^{1}$ :

$$
\begin{aligned}
x_{k} & =\bar{A}\left(\varsigma_{k}\right) x_{k-1}+\bar{H}\left(\varsigma_{k}\right) y_{k}+\bar{W}_{1}\left(\varsigma_{k}\right) w_{k-1}+\bar{W}_{2}\left(\varsigma_{k}\right) w_{k} \\
& =\sum_{i=1}^{\bar{M}} \rho_{i}\left(\varsigma_{k}\right)\left[\bar{A}^{i} x_{k-1}+\bar{H}^{i} y_{k}+\bar{W}_{1}^{i} w_{k-1}+\bar{W}_{2}^{i} w_{k}\right] \\
y_{k} & =\check{C}\left(\varsigma_{k}\right) x_{k}+\check{W}_{2}\left(\varsigma_{k}\right) w_{k}=\sum_{i=1}^{\bar{M}} \rho_{i}\left(\varsigma_{k}\right)\left[\check{C}^{i} x_{k}+\check{W}_{2}^{i} w_{k}\right]
\end{aligned}
$$

\footnotetext{
${ }^{1}$ Notice that the premise variables denoted by $\varsigma_{k}$ are not the same as $s_{k}$. Also, the matrices $\check{C}^{i}, \check{W}_{2}^{i}, i=1, \ldots, \bar{M}$ are different from the matrices $C^{i}, W_{2}^{i}, i=1, \ldots, M$.
} 
with:

$$
\rho_{i}\left(\varsigma_{k}\right) \geq 0 \quad \forall i=1, \ldots, \bar{M} \quad \sum_{i=1}^{\bar{M}} \rho_{i}\left(\varsigma_{k}\right)=1
$$

Then, the following UIO is proposed for the system (35)-(36):

$$
\begin{gathered}
\hat{x}_{k}=\bar{A}\left(\varsigma_{k}\right) \hat{x}_{k-1}+\bar{H}\left(\varsigma_{k}\right) y_{k}+K\left(\varsigma_{k}\right)\left(y_{k-1}-\hat{y}_{k-1}\right) \\
=\sum_{i=1}^{\bar{M}} \rho_{i}\left(\varsigma_{k}\right)\left[\bar{A}^{i} \hat{x}_{k-1}+\bar{H}^{i} y_{k}+K^{i}\left(y_{k-1}-\hat{y}_{k-1}\right)\right] \\
\hat{y}_{k}=\check{C}\left(\varsigma_{k}\right) \hat{x}_{k}=\sum_{i=1}^{\bar{M}} \rho_{i}\left(\varsigma_{k}\right) \check{C}^{i} \hat{x}_{k}
\end{gathered}
$$

Example: Let us consider a TS system as in (1)-(2), with:

$$
\begin{aligned}
& A\left(s_{k}\right)=\left(\begin{array}{ccc}
-0.4-0.1 s_{k} & 0.2+0.2 s_{k} & 0.3-0.1 s_{k} \\
0.3-0.2 s_{k} & -0.6+0.2 s_{k} & 0.3-0.3 s_{k} \\
0.4+0.4 s_{k} & 0.2+0.5 s_{k} & 0.6-s_{k}
\end{array}\right) \quad B=\left(\begin{array}{l}
1 \\
2 \\
1
\end{array}\right) \quad C\left(s_{k}\right)=\left(\begin{array}{ccc}
1 & 0-1 \\
1+s_{k} & 2 & 1
\end{array}\right) \\
& W_{1}\left(s_{k}\right)=\left(\begin{array}{ccccc}
1+s_{k} & 0 & 0 & 0 & 0 \\
0 & 1 & 0 & 0 & 0 \\
0 & 0 & 1 & 0 & 0
\end{array}\right) \quad W_{2}\left(s_{k}\right)=\left(\begin{array}{ccccc}
0 & 0 & 0 & 1+s_{k} & 0 \\
0 & 0 & 0 & 0 & 1
\end{array}\right)
\end{aligned}
$$

and $s_{k} \in[0,1]$. It is straightforward to check that:

$$
C\left(s_{k+1}\right) B=\left(\begin{array}{c}
0 \\
6+s_{k+1}
\end{array}\right)
$$

has rank 1, i.e. the same rank of $B, \forall s_{k+1} \in[0,1]$, such that (18) holds and it is possible to calculate $H\left(s_{k}, s_{k+1}\right)$ using (28), that leads to:

$$
H\left(s_{k}, s_{k+1}\right)=\left(0 \frac{1}{6+s_{k+1}}\right)
$$

Hence, the matrices $\bar{A}\left(s_{k-1}, s_{k}\right), \bar{H}\left(s_{k-1}, s_{k}\right), \bar{W}_{1}\left(s_{k-1}, s_{k}\right)$ and $\bar{W}_{2}\left(s_{k-1}, s_{k}\right)$, calculated as (31)(34), take the following form:

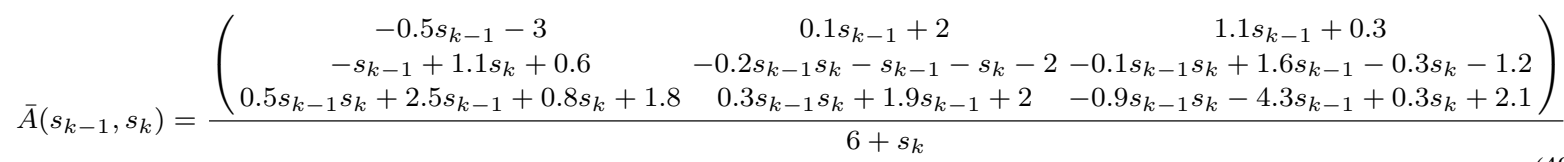

$$
\bar{H}\left(s_{k-1}, s_{k}\right)=\frac{\left(\begin{array}{ll}
0 & 1 \\
0 & 2 \\
0 & 1
\end{array}\right)}{6+s_{k}}
$$




$$
\bar{W}_{1}\left(s_{k-1}, s_{k}\right)=\frac{\left(\begin{array}{ccccc}
5+5 s_{k-1} & -2 & -1 & 0 & 0 \\
-2 s_{k-1} s_{k} & 2+s_{k} & -2 & 0 & 0 \\
-s_{k-1} s_{k}-s_{k-1}-s_{k}-1 & -2 & 5+s_{k} & 0 & 0
\end{array}\right)}{6+s_{k}}
$$

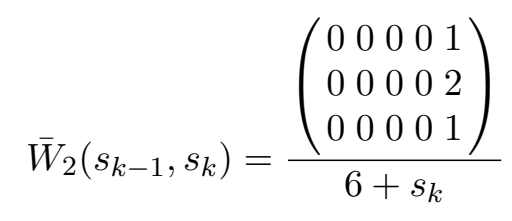

By defining the new premise variables $\varsigma_{1}=s_{k-1}, \varsigma_{2}=s_{k}$ and $\varsigma_{3}=\frac{1}{6+s_{k}}$, and considering that $\varsigma_{1} \in[0,1], \varsigma_{2} \in[0,1]$ and $\varsigma_{3} \in[1 / 7,1 / 6],(35)$-(36) becomes a set of $\bar{M}=8$ subsystems, with the following matrices

$$
\begin{aligned}
& \bar{A}^{1}=\left(\begin{array}{ccc}
-0.4286 & 0.2857 & 0.0429 \\
0.0857 & -0.2857 & -0.1714 \\
0.2571 & 0.2857 & 0.3000
\end{array}\right) \quad \bar{A}^{2}=\left(\begin{array}{ccc}
-0.5000 & 0.3333 & 0.0500 \\
0.1000 & -0.3333 & -0.2000 \\
0.3000 & 0.3333 & 0.3500
\end{array}\right) \\
& \bar{A}^{3}=\left(\begin{array}{ccc}
-0.4286 & 0.2857 & 0.0429 \\
0.2429 & -0.4286 & -0.2143 \\
0.3714 & 0.2857 & 0.3429
\end{array}\right) \quad \bar{A}^{4}=\left(\begin{array}{ccc}
-0.5000 & 0.3333 & 0.0500 \\
0.2833 & -0.5000 & -0.2500 \\
0.4333 & 0.3333 & 0.4000
\end{array}\right) \\
& \bar{A}^{5}=\left(\begin{array}{ccc}
-0.5000 & 0.3000 & 0.2000 \\
-0.0571 & -0.4286 & 0.0571 \\
0.6143 & 0.5571 & -0.3143
\end{array}\right) \quad \bar{A}^{6}=\left(\begin{array}{ccc}
-0.5833 & 0.3500 & 0.2333 \\
-0.0667 & -0.5000 & 0.0667 \\
0.7167 & 0.6500 & -0.3667
\end{array}\right) \\
& \bar{A}^{7}=\left(\begin{array}{ccc}
-0.5000 & 0.3000 & 0.2000 \\
0.1000 & -0.6000 & 0 \\
0.8000 & 0.6000 & -0.4000
\end{array}\right) \quad \bar{A}^{8}=\left(\begin{array}{ccc}
-0.5833 & 0.3500 & 0.2333 \\
0.1167 & -0.7000 & 0 \\
0.9333 & 0.7000 & -0.4667
\end{array}\right) \\
& \bar{H}^{1}=\bar{H}^{3}=\bar{H}^{5}=\bar{H}^{7}=\left(\begin{array}{ll}
0 & 0.1429 \\
0 & 0.2857 \\
0 & 0.1429
\end{array}\right) \quad \bar{H}^{2}=\bar{H}^{4}=\bar{H}^{6}=\bar{H}^{8}=\left(\begin{array}{ll}
0 & 0.1667 \\
0 & 0.3333 \\
0 & 0.1667
\end{array}\right)
\end{aligned}
$$

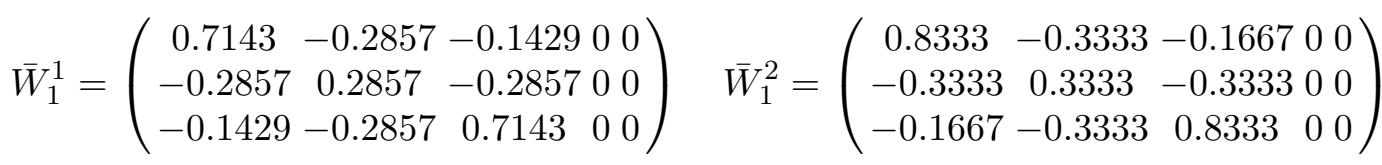

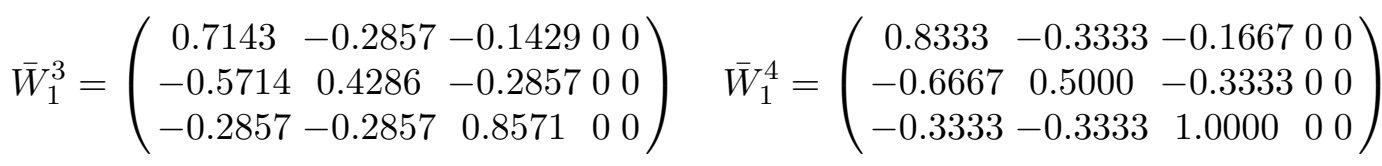




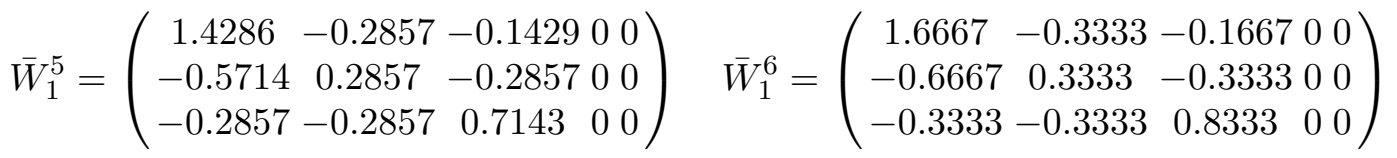

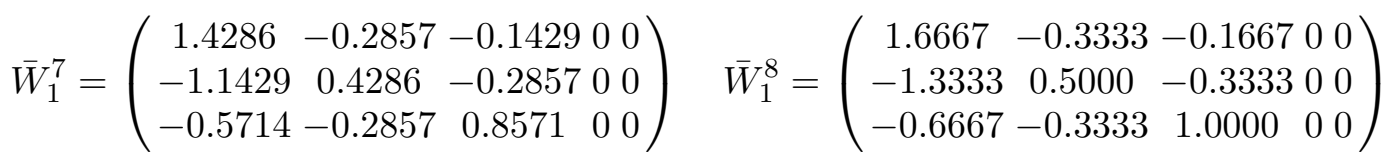

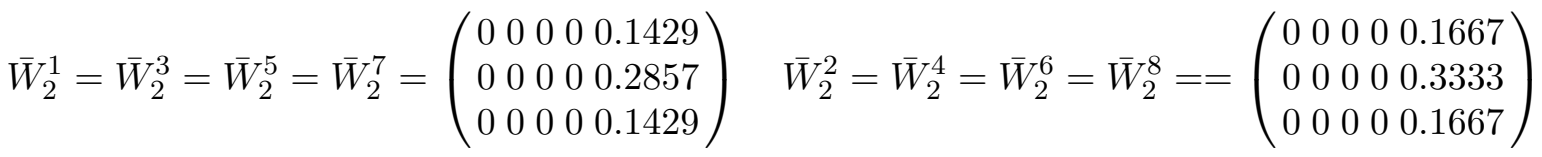

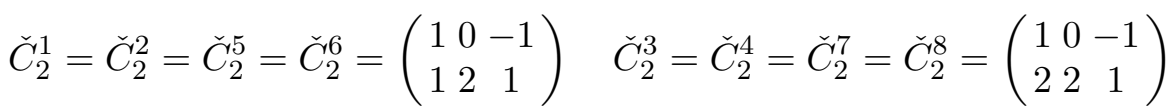

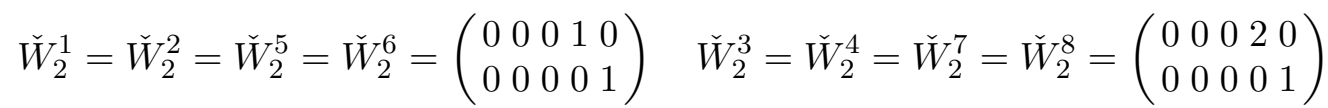

\subsection{Design procedure}

As it was mentioned, instead of eliminating the effect of $w_{k}$, its influence on the performance of the UIO will be minimized.

The objective is to design the observer (38) in such a way that the state estimation error $e_{k}$ is asymptotically convergent and the following upper bound is guaranteed:

$$
\|e\|_{l_{2}} \leq \omega\|w\|_{l_{2}}
$$

where $\omega>0$ is a prescribed disturbance attenuation level.

Noticing that (38) can be written as:

$$
\hat{x}_{k}=\sum_{i=1}^{\bar{M}} \rho_{i}\left(\varsigma_{k}\right) \sum_{j=1}^{\bar{M}} \rho_{j}\left(\varsigma_{k-1}\right)\left[\bar{A}^{i} \hat{x}_{k-1}+\bar{H}^{i} y_{k}+K^{i}\left(\check{C}^{j} x_{k-1}+\check{W}_{2}^{j} w_{k-1}-\check{C}^{j} \hat{x}_{k-1}\right)\right]
$$

and it can be shown that the state estimation error obeys the following equation:

$$
e_{k}=\sum_{i=1}^{\bar{M}} \rho_{i}\left(\varsigma_{k}\right) \sum_{j=1}^{\bar{M}} \rho_{j}\left(\varsigma_{k-1}\right)\left[A_{1}^{i, j} e_{k-1}+\tilde{W}_{1}^{i, j} w_{k-1}+\bar{W}_{2}^{i} w_{k}\right]
$$

where:

$$
A_{1}^{i, j}=\bar{A}^{i}-K^{i} \check{C}^{j}, \quad \tilde{W}_{1}^{i, j}=\bar{W}_{1}^{i}-K^{i} \check{W}_{2}^{j}
$$

In the observer design approaches presented in the literature (cf. Li and Fu (1997); Zemouche et al. (2008)), the state estimation error depends on $w_{k}$ only, while (46) contains both $w_{k-1}$ and $w_{k}$. This situation requires a different design approach that is given by the following theorem.

Theorem 3.1. For a prescribed disturbance attenuation level $\omega>0$ for the state estimation error (46), the $\mathcal{H}_{\infty}$ observer design problem for the system (35)-(36) and the observer (38)-(39) is solvable if there 
exist matrices $P^{i} \succ 0, N^{i}(i=1, \ldots, \bar{M})$ and $U$ such that the following inequality is satisfied for all $i, j, l=1, \ldots, \bar{M}$ :

$$
\Upsilon_{i, j}^{l}=\left[\begin{array}{cccc}
I-P^{i} & 0 & 0 & \left(A_{1}^{i, j}\right)^{T} U^{T} \\
0 & -\mu^{2} I & 0 & \left(\tilde{W}_{1}^{i, j}\right)^{T} U^{T} \\
0 & 0 & -\mu^{2} I & \left(\bar{W}_{2}^{i}\right)^{T} U^{T} \\
U A_{1}^{i, j} & U \tilde{W}_{1}^{i, j} & U \bar{W}_{2}^{i} & P^{l}-U-U^{T}
\end{array}\right] \prec 0
$$

with $\mu=\omega / \sqrt{2}$ and:

$$
\begin{array}{r}
U A_{1}^{i, j}=U \bar{A}^{i}-U K^{i} \check{C}^{j}=U \bar{A}^{i}-N^{i} \check{C}^{j} \\
U \tilde{W}_{1}^{i, j}=U \bar{W}_{1}^{i}-U K^{i} \check{W}_{2}^{j}=U \bar{W}_{1}^{i}-N^{i} \check{W}_{2}^{j} .
\end{array}
$$

Proof. The problem of $\mathcal{H}_{\infty}$ observer design ( $\mathrm{Li}$ and Fu 1997; Zemouche et al. 2008) is to determine the matrices $K^{i}, i=1, \ldots, \bar{M}$ such that

$$
\begin{aligned}
& \lim _{k \rightarrow \infty} e_{k}=0 \quad \text { for } w_{k}=0 \quad \forall k \\
& \|e\|_{l_{2}} \leq \omega\|w\|_{l_{2}} \text { for } w_{k} \neq 0, e_{0}=0
\end{aligned}
$$

In this paper, a quadratic Lyapunov approach is used, which means that it is sufficient to find a function $V_{k} \succ 0$ such that:

$$
\Delta V_{k-1}+e_{k-1}^{T} e_{k-1}-\mu^{2} w_{k-1}^{T} w_{k-1}-\mu^{2} w_{k}^{T} w_{k}<0, \quad k=1, \ldots, \infty
$$

where $\Delta V_{k-1}=V_{k}-V_{k-1}, \mu>0$. Indeed, if $w_{k}=0, k=1, \ldots, \infty$, then (53) can be expressed as:

$$
\Delta V_{k-1}+e_{k-1}^{T} e_{k-1}<0, k=1, \ldots \infty
$$

and hence $\Delta V_{k-1}<0$, which leads to (51). If $w_{k} \neq 0, k=1, \ldots, \infty$, then (53) yields:

$$
J=\sum_{k=1}^{\infty}\left(\Delta V_{k-1}+e_{k-1}^{T} e_{k-1}-\mu^{2} w_{k-1}^{T} w_{k-1}-\mu^{2} w_{k}^{T} w_{k}\right)<0
$$

which can be written as:

$$
J=-V_{0}+\sum_{k=1}^{\infty} e_{k-1}^{T} e_{k-1}-\mu^{2} \sum_{k=1}^{\infty} w_{k-1}^{T} w_{k-1}-\mu^{2} \sum_{k=1}^{\infty} w_{k}^{T} w_{k}<0
$$

Bearing in mind that:

$$
\mu^{2} \sum_{k=1}^{\infty} w_{k}^{T} w_{k}=\mu^{2} \sum_{k=1}^{\infty} w_{k-1}^{T} w_{k-1}-\mu^{2} w_{0}^{T} w_{0}
$$

the inequality (56) can be written as:

$$
J=-V_{0}+\sum_{k=1}^{\infty} e_{k-1}^{T} e_{k-1}-2 \mu^{2} \sum_{k=1}^{\infty} w_{k-1}^{T} w_{k-1}+\mu^{2} w_{0}^{T} w_{0}<0
$$

Knowing that $V_{0}=0$ for $e_{0}=0$, (58) leads to (52) with $\omega=\sqrt{2} \mu$. 
Let us consider the following form for the Lyapunov function:

$$
V_{k}=\sum_{i=1}^{\bar{M}} \rho_{i}\left(\varsigma_{k}\right) e_{k}^{T} P^{i} e_{k}, \quad P^{i} \succ 0
$$

Thus, by defining $v_{k-1}=\left[e_{k-1}^{T}, w_{k-1}^{T}, w_{k}^{T}\right]$, it can be shown that the condition (53) is equivalent to:

$$
\sum_{i=1}^{\bar{M}} \rho_{i}\left(\varsigma_{k-1}\right) \sum_{j=1}^{\bar{M}} \rho_{j}\left(\varsigma_{k}\right) \sum_{l=1}^{\bar{M}} \rho_{l}\left(\varsigma_{k}\right) v_{k-1}^{T} \Phi_{i, j}^{l} v_{k-1}<0
$$

where:

$$
\Phi_{i, j}^{l}=\left[\begin{array}{ccc}
\left(A_{1}^{i, j}\right)^{T} P^{l} A_{1}^{i, j}+I-P^{i} & \left(A_{1}^{i, j}\right)^{T} P^{l} \tilde{W}_{1}^{i, j} & \left(A_{1}^{i, j}\right)^{T} P^{l} \bar{W}_{2}^{i} \\
\left(\tilde{W}_{1}^{i, j}\right)^{T} P^{l} A_{1}^{i, j} & \left(\tilde{W}_{1}^{i, j}\right)^{T} P^{l} \tilde{W}_{1}^{i, j}-\mu^{2} I & \left(\tilde{W}_{1}^{i, j}\right)^{T} P^{l} \bar{W}_{2}^{i} \\
\left(\bar{W}_{2}^{i}\right)^{T} P^{l} A_{1}^{i, j} & \left(\bar{W}_{2}^{i}\right)^{T} P^{l} \tilde{W}_{1}^{i, j} & \left(\bar{W}_{2}^{i}\right)^{T} P^{l} \bar{W}_{2}^{i}-\mu^{2} I
\end{array}\right]
$$

Let us remind the following lemma (de Oliveira et al. 1999):

Lemma 3.1. The following statements are equivalent:

(1) There exists $X \succ O$ such that

$$
Y^{T} X Y-W \prec O
$$

(2) There exists $X \succ O$ such that

$$
\left[\begin{array}{cc}
-W & Y^{T} U^{T} \\
U Y & X-U-U^{T}
\end{array}\right] \prec O .
$$

Applying Lemma 3.1 to (61) leads to (48), which completes the proof.

Notice that the inequalities (48) involve a multiple sum negativity problem. As remarked by Guerra et al. (2009), many works in the literature have tried to reduce the pessimism of these conditions (Wang et al. 1996). Among the proposed solutions, the Lemma proposed in Tuan et al. (2001) is a good compromise between complexity and conservatism, which in the case of (48) leads to the following:

Corollary 3.1. Condition (48) is fulfilled provided that the following conditions hold:

$$
\begin{gathered}
\Upsilon_{i, i}^{l} \prec 0, \quad i \in\{1, \ldots, \bar{M}\} \\
\frac{2}{M-1} \Upsilon_{i, i}^{l}+\Upsilon_{i, j}^{l}+\Upsilon_{j, i}^{l} \prec 0, \quad i, j, l \in\{1, \ldots, \bar{M}\}, \quad i \neq j
\end{gathered}
$$

Finally, the design procedure consists in solving (64)-(65) with respect to $U, N^{i}$ and $P^{i}$ ( $i=$ $1, \ldots, \bar{M})$, and then calculating:

$$
K^{i}=U^{-1} N^{i}, \quad i=1, \ldots, \bar{M}
$$

Remark 3.1. When $\mu \rightarrow+\infty$, (48) reduces to:

$$
\Upsilon_{i, j}^{l}=\left[\begin{array}{cc}
P^{i}-I & \left(A_{1}^{i, j}\right)^{T} U^{T} \\
U A_{1}^{i, j} & U+U^{T}-P^{l}
\end{array}\right] \succ 0
$$

Hence, a necessary and sufficient condition for the existence of $\omega>0$ such that the $\mathcal{H}_{\infty}$ observer design problem for the system (35)-(36) and the observer (38)-(39) is solvable, is that there exist 
matrices $P^{i} \succ 0, N^{i}(i=1, \ldots, \bar{M})$ and $U$ such that (67) is satisfied for all $i, j, l=1, \ldots, \bar{M}$. Notice that (67) represents a stability condition (de Oliveira et al. 1999), that can be satisfied by an appropriate choice of the gains $K^{i}$ only if the pairs $\left(\bar{A}^{i}, \check{C}^{j}\right)$ are detectable for all $i, j=1, \ldots, \bar{M}$ (necessary but not sufficient condition).

\subsection{Guaranteed decay rate}

The proposed procedure can be used to design a fault identification scheme in such a way that a prescribed disturbance attenuation level is achieved with respect to the state estimation error while guaranteeing the convergence of the observer. It was shown that when $w_{k}=0$, then the state estimation error converges asymptotically to zero. It is important to note that the proposed observer-based fault identification scheme can also be used for control purposes within the FTC framework (Mahmoud et al. 2003; Noura et al. 2009; Ducard 2009; Puig 2010). Thus, a requirement stronger than asymptotic stability, such as a guaranteed decay rate of the state estimation error, could be needed.

Corollary 3.2. For a prescribed disturbance attenuation level $\omega>0$ and a prescribed guaranteed decay rate $\theta>0$ for the state estimation error (46), the $\mathcal{H}_{\infty}$ observer design problem for the system (35)-(36) and the observer (38)-(39) is solvable if there exist matrices $P^{i} \succ 0, N^{i}(i=1, \ldots, \bar{M})$ and $U$ such that the following inequality is satisfied for all $i, j, l=1, \ldots, \bar{M}$ :

$$
\Upsilon_{i, j}^{l}=\left[\begin{array}{cccc}
I-\tau P^{i} & 0 & 0 & A_{1}^{i, j} U^{T} \\
0 & -\mu^{2} I & 0 & \tilde{W}_{1}^{i, j} U^{T} \\
0 & 0 & -\mu^{2} I & \bar{W}_{2}^{i} U^{T} \\
U A_{1}^{i, j} & U \tilde{W}_{1}^{i, j} & U \bar{W}_{2}^{i} & P^{l}-U-U^{T}
\end{array}\right]
$$

with $\tau=e^{-2 \theta}>0, \mu=\omega / \sqrt{2}$ and $U A_{1}^{i, j}, U \tilde{W}_{1}^{i, j}$ defined as in (49)-(91).

Proof. Following Abbaszadeh and Marquez (2009), for $w_{k}=0(k=0, \ldots, \infty)$ and $\theta>0$, the decay rate of the state estimation error is defined as:

$$
\lim _{k \rightarrow \infty} e^{\theta k}\left\|e_{k}\right\|=0
$$

Suppose that the following holds for the quadratic Lyapunov function $V_{k}$ introduced in the proof of Theorem 3.1:

$$
\Delta V_{k-1}=V_{k}-V_{k-1} \leq-\left(1-e^{-2 \theta}\right) V_{k-1}
$$

which is equivalent to:

$$
V_{k} \leq e^{-2 \theta k} V_{0}
$$

Bearing in mind that:

$$
\lambda_{\min }\left(P_{k}\right) e_{k}^{T} e_{k} \leq e_{k}^{T} P_{k} e_{k} \leq \lambda_{\max }\left(P_{k}\right) e_{k}^{T} e_{k},
$$

with:

$$
P_{k}=\sum_{i=1}^{\bar{M}} \rho_{i}\left(\varsigma_{k}\right) P^{i}
$$

while $\lambda_{\min }\left(P_{k}\right)$ and $\lambda_{\max }\left(P_{k}\right)$ stand for the minimum and maximum eigenvalues of $P_{k}$, respectively, it 
can be shown that:

$$
\left\|e_{k}\right\| \leq \sqrt{\frac{\lambda_{\max }\left(P_{0}\right)}{\lambda_{\min }\left(P_{k}\right)}} e^{-\theta k}\left\|e_{0}\right\|,
$$

which is equivalent to (69).

Thus, using (70), to attain a guaranteed decay rate $\theta$ it is needed that:

$$
\Delta V_{k-1} \leq-\left(1-e^{-2 \theta}\right) V_{k}=-\left(1-e^{-2 \theta}\right) e_{k}^{T} P_{k} e_{k},
$$

which is equivalent to replacing $-P^{i}$ in (48) by $-\tau P^{i}\left(\tau=e^{-2 \theta}\right.$, i.e. $\left.\theta=-\frac{1}{2} \ln (\tau)\right)$, which gives (68), completing the proof.

Hence, given a prescribed disturbance attenuation level $\omega$, the following optimisation problem can be formulated:

$$
\tau^{*}=\min _{\tau>0, P^{i} \succ 0, N^{i}, U} \tau
$$

subject to (64) and (65).

An alternative solution is to minimize $\mu$ and $\tau$ simultaneously, i.e., given a scalar $0 \leq \lambda \leq 1$ the optimization problem is (cf. Abbaszadeh and Marquez (2009)):

$$
\left(\tau^{*}, \mu^{*}\right)=\min _{\tau>0, \mu, P^{i} \succ 0, N^{i}, U} \lambda \tau+(1-\lambda) \mu
$$

subject to (64) and (65).

These minimization problems involve bilinear matrix inequalities (BMIs) due to the products of $\tau$ by $P^{i}$. However, a line search approach allows to solve it using LMI solvers.

\subsection{Fault estimation strategy}

Since the design procedure of the UIO is provided, it is possible to propose a fault estimation strategy that is based on the obtained state estimates. For that purpose, let us rewrite the system (1)-(2) as follows:

$$
\begin{gathered}
x_{k+1}=A\left(s_{k}\right) x_{k}+B\left(s_{k}\right) u_{k}+B\left(s_{k}\right) f_{k}+W_{1}\left(s_{k}\right) w_{k} \\
y_{k+1}=C\left(s_{k+1}\right) x_{k+1}+W_{2}\left(s_{k+1}\right) w_{k+1}
\end{gathered}
$$

Taking into account that the rank condition (18) holds, it is possible to calculate the matrix $H\left(s_{k}, s_{k+1}\right)$, as in (28). Then, pre-multiplying (79) by $H\left(s_{k}, s_{k+1}\right)$ and replacing in it (78), the following is obtained:

$$
f_{k}=H\left(s_{k}, s_{k+1}\right)\left[y_{k+1}-C\left(s_{k+1}\right) A\left(s_{k}\right) x_{k}-C\left(s_{k+1}\right) W_{1}\left(s_{k}\right) w_{k}-W_{2}\left(s_{k+1}\right) w_{k+1}\right]-u_{k} .
$$

Thus, the fault estimate is given by:

$$
\hat{f}_{k}=H\left(s_{k}, s_{k+1}\right)\left[y_{k+1}-C\left(s_{k+1}\right) A\left(s_{k}\right) \hat{x}_{k}\right]-u_{k} .
$$

\subsection{Observer design with unmeasurable premise variables}

Let us consider the case where the premise variables are partially or completely unmeasured. In this case, the assumption that $\check{C}^{i}=\check{C}$ and $\check{W}_{2}^{i}=\check{W}_{2}, i=1, \ldots, \bar{M}$, is done. Then, the UIO (38)-(39) is 
slightly modified, as follows:

$$
\begin{gathered}
\hat{x}_{k}=\bar{A}\left(\hat{\varsigma}_{k}\right) \hat{x}_{k-1}+\bar{H}\left(\hat{\varsigma}_{k}\right) y_{k}+K\left(\hat{\varsigma}_{k}\right)\left(y_{k-1}-\hat{y}_{k-1}\right) \\
=\sum_{i=1}^{\bar{M}} \rho_{i}\left(\hat{\varsigma}_{k}\right)\left[\bar{A}^{i} \hat{x}_{k-1}+\bar{H}^{i} y_{k}+K^{i}\left(y_{k-1}-\hat{y}_{k-1}\right)\right] \\
\hat{y}_{k}=\check{C} \hat{x}_{k}
\end{gathered}
$$

where $\hat{\varsigma}_{k}$ is an estimation of $\varsigma_{k}$.

Then, taking into account (1)-(2), the estimation error obeys the following equation:

$$
\begin{aligned}
e_{k} & =\left(\bar{A}\left(\hat{\varsigma}_{k}\right)-K\left(\hat{\varsigma}_{k}\right) \check{C}\right) e_{k-1}+\left(\bar{W}_{1}\left(\hat{\varsigma}_{k}\right)-K\left(\hat{\varsigma}_{k}\right) \check{W}_{2}\right) w_{k-1}+\bar{W}_{2}\left(\hat{\varsigma}_{k}\right) w_{k}+\Delta(\cdot) \\
& =\sum_{i=1}^{\bar{M}} \rho_{i}\left(\hat{\varsigma}_{k}\right)\left[A_{1}^{i} e_{k-1}+\tilde{W}_{1}^{i} w_{k-1}+\bar{W}_{2}^{i} w_{k}\right]+\Delta(\cdot)
\end{aligned}
$$

where:

$$
A_{1}^{i}=\bar{A}^{i}-K^{i} \check{C}, \quad \tilde{W}_{1}^{i}=\bar{W}_{1}^{i}-K^{i} \check{W}_{2}
$$

and $\Delta(\cdot)$ is given by:

$$
\begin{aligned}
\Delta(\cdot) & =\left(\bar{A}\left(\varsigma_{k}\right)-\bar{A}\left(\varsigma_{k}\right)\right) x_{k-1}+\left(\bar{H}\left(\varsigma_{k}\right)-\bar{H}\left(\hat{\varsigma}_{k}\right)\right) y_{k}+\left(\bar{W}_{1}\left(\varsigma_{k}\right)-\bar{W}_{1}\left(\hat{\varsigma}_{k}\right) w_{k-1}+\left(\bar{W}_{2}\left(\varsigma_{k}\right)-\bar{W}_{2}\left(\hat{\varsigma}_{k}\right) w_{k}\right.\right. \\
& =\sum_{i=1}^{\bar{M}}\left(\rho_{i}\left(\varsigma_{i}\right)-\rho_{i}\left(\hat{\varsigma}_{i}\right)\right)\left[\bar{A}^{i} x_{k-1}+\bar{H}^{i} y_{k}+\bar{W}_{1}^{i} w_{k-1}+\bar{W}_{2}^{i} w_{k}\right]
\end{aligned}
$$

Assumption 1: $\Delta(\cdot)$ is one-sided Lipschitz (Zhang et al. 2012) in $e_{k}$, i.e. there exists $\rho \in \mathrm{R}$ such that, for an arbitrary $e_{k} \in \mathrm{R}^{n}$ :

$$
\left\langle\Delta(\cdot), e_{k}\right\rangle \leq \rho\left\|e_{k}\right\|^{2}
$$

where $\langle\cdot, \cdot\rangle$ denotes the inner product, and $\rho \in \mathrm{R}^{n}$ is called the one-sided Lipschitz constant, which can be positive, zero, or even negative.

Assumption 2: $\Delta(\cdot)$ is quadratic inner-bounded (Zhang et al. 2012) in $e_{k-1}$, i.e. for an arbitrary $e_{k-1} \in$ $\mathrm{R}^{n}$, there exist $\sigma, \varphi \in \mathrm{R}$ such that:

$$
\Delta(\cdot)^{T} \Delta(\cdot) \leq \sigma\left\|e_{k-1}\right\|^{2}+\varphi\left\langle e_{k-1}, \Delta(\cdot)\right\rangle
$$

Notice that the properties of one-sided Lipschitz and quadratic inner-boundedness include the Lipschitz property as a particular case ${ }^{2}$.

The discussion in Zhang et al. (2012) has shown that, under the setting of Lipschitz nonlinearities, it is difficult to design observers in the case of Lipschitz constant bigger than one. This fact has motivated the observer design for one-sided Lipschitz systems, with the aim of reducing the conservatism of the results.

Corollary 3.3. Assume that $\Delta(\cdot)$, defined as in (86), satisfies conditions (87)-(88) with constants $\rho, \sigma$ and $\varphi$. Then, for a prescribed disturbance attenuation level $\omega>0$ and a prescribed guaranteed decay

\footnotetext{
${ }^{2}$ In practice, it is not required that these properties hold $\forall e_{k-1}, e_{k} \in \mathrm{R}^{n}$, but only for values that are reasonable taking into account the system's characteristics and sensors.
} 
rate $\theta>0$ for the state estimation error (84), the $\mathcal{H}_{\infty}$ observer design problem for the system (35)-(36) with $\check{C}^{i}=\check{C}$ and $\check{W}_{2}^{i}=\check{W}_{2}, i=1, \ldots, \bar{M}$, and the observer (82)-(83) is solvable if there exist matrices $P^{i} \succ 0, N^{i}(i=1, \ldots, \bar{M})$ and $U$ such that the following inequality is satisfied for all $i, l=1, \ldots, \bar{M}$ :

$$
\Upsilon_{i, j}^{l}=\left[\begin{array}{ccccc}
I-\tau P^{i}+\varepsilon_{1} \rho I+\varepsilon_{2} \sigma I & 0 & 0 & \frac{\varphi \varepsilon_{2}-\varepsilon_{1}}{2} I & A_{1}^{i} U^{T} \\
0 & -\mu^{2} I & 0 & 0 & \tilde{W}_{1}^{i} U^{T} \\
0 & 0 & -\mu^{2} I & 0 & \bar{W}_{2}^{i} U^{T} \\
\frac{\varphi \varepsilon_{2}-\varepsilon_{1}}{2} I & 0 & 0 & -\varepsilon_{2} I & U^{T} \\
U A_{1}^{i} & U \tilde{W}_{1}^{i} & U \bar{W}_{2}^{i} & U & P^{l}-U-U^{T}
\end{array}\right]
$$

with $\tau=e^{-2 \theta}>0, \mu=\omega / \sqrt{2}$ and $U A_{1}^{i}, U \tilde{W}_{1}^{i}$ defined as:

$$
\begin{array}{r}
U A_{1}^{i}=U \bar{A}^{i}-U K^{i} \check{C}=U \bar{A}^{i}-N^{i} \check{C} \\
U \tilde{W}_{1}^{i}=U \bar{W}_{1}^{i}-U K^{i} \check{W}_{2}=U \bar{W}_{1}^{i}-N^{i} \check{W}_{2} .
\end{array}
$$

Proof. Let us consider the following Lyapunov function:

$$
V_{k}=\sum_{i=1}^{\bar{M}} \rho_{i}\left(\hat{\varsigma}_{k}\right) e_{k}^{T} P^{i} e_{k}, \quad P^{i} \succ 0
$$

and let us assume that (70) holds (see proof of Corollary 3.2). Then, by defining $v_{k-1}=$ $\left[e_{k-1}^{T}, w_{k-1}^{T}, w_{k}^{T}, \Delta(\cdot)^{T}\right]$, it can be shown that the condition (70) is equivalent to:

$$
\sum_{i=1}^{\bar{M}} \rho_{i}\left(\hat{\varsigma}_{k-1}\right) \sum_{l=1}^{\bar{M}} \rho_{l}\left(\hat{\varsigma}_{k}\right) v_{k-1}^{T} \Phi_{i}^{l} v_{k-1}<0
$$

where:

$$
\Phi_{i}^{l}=\left[\begin{array}{cccc}
\left(A_{1}^{i}\right)^{T} P^{l} A_{1}^{i}+I-\tau P^{i} & \left(A_{1}^{i}\right)^{T} P^{l} \tilde{W}_{1}^{i} & \left(A_{1}^{i}\right)^{T} P^{l} \bar{W}_{2}^{i} & \left(A^{i}\right)^{T} P^{l} \\
\left(\tilde{W}_{1}^{i}\right)^{T} P^{l} A_{1}^{i} & \left(\tilde{W}_{1}^{i}\right)^{T} P^{l} \tilde{W}_{1}^{i}-\mu^{2} I & \left(\tilde{W}_{1}^{i}\right)^{T} P^{l} \bar{W}_{2}^{i} & \left(\tilde{W}_{1}^{i}\right)^{T} P^{l} \\
\left(\bar{W}_{2}^{i}\right)^{T} P^{l} A_{1}^{i} & \left(\bar{W}_{2}^{i}\right)^{T} P^{l} \tilde{W}_{1}^{i} & \left(\bar{W}_{2}^{i}\right)^{T} P^{l} \bar{W}_{2}^{i}-\mu^{2} I\left(\bar{W}_{2}^{i}\right)^{T} P^{l} \\
P^{l} A_{1}^{i} & P^{l} \tilde{W}_{1}^{i} & P^{l} \bar{W}_{2}^{i} & P^{l}
\end{array}\right]
$$

From (87), we get $\rho e_{k}^{T} e_{k}-e_{k}^{T} \Delta(\cdot) \geq 0$. Therefore, for any $\varepsilon_{1}>0$ :

$$
\varepsilon_{1}\left[\begin{array}{c}
e_{k-1} \\
\Delta(\cdot)
\end{array}\right]^{T}\left[\begin{array}{cc}
\rho I & -\frac{I}{2} \\
-\frac{I}{2} & -I
\end{array}\right]\left[\begin{array}{c}
e_{k-1} \\
\Delta(\cdot)
\end{array}\right] \geq 0
$$

Similarly, from (88), we have for any $\varepsilon_{2}>0$ :

$$
\varepsilon_{2}\left[\begin{array}{c}
e_{k-1} \\
\Delta(\cdot)
\end{array}\right]^{T}\left[\begin{array}{cc}
\sigma I & \frac{\varphi I}{2} \\
\frac{\varphi I}{2} & -I
\end{array}\right]\left[\begin{array}{c}
e_{k-1} \\
\Delta(\cdot)
\end{array}\right] \geq 0
$$

By combining (93)-(94) with (95)-(96), the following is obtained:

$$
\Phi_{i}^{l}=\left[\begin{array}{cccc}
\left(A_{1}^{i}\right)^{T} P^{l} A_{1}^{i}+I-\tau P^{i}+\varepsilon_{1} \rho I+\varepsilon_{2} \sigma I & \left(A_{1}^{i}\right)^{T} P^{l} \tilde{W}_{1}^{i} & \left(A_{1}^{i}\right)^{T} P^{l} \bar{W}_{2}^{i} & \left(A_{1}^{i}\right)^{T} P^{l}+\frac{\varphi \varepsilon_{2}-\varepsilon_{1}}{} I \\
\left(\tilde{W}_{1}^{i}\right)^{T} P^{l} A_{1}^{i} & \left(\tilde{W}_{1}^{i}\right)^{T} P^{l} \tilde{W}_{1}^{i}-\mu^{2} I & \left(\tilde{W}_{1}^{i}\right)^{T} P^{l} \bar{W}_{2}^{i} & \left(\tilde{W}_{1}^{i}\right)^{T} P^{l}{ }^{2} \\
\left(\bar{W}_{2}^{i}\right)^{T} P^{l} A_{1}^{i} & \left(\bar{W}_{2}^{i}\right)^{T} P^{l} \tilde{W}_{1}^{i} & \left(\bar{W}_{2}^{i}\right)^{T} P^{l} \bar{W}_{2}^{i}-\mu^{2} I & \left(\bar{W}_{2}^{i}\right)^{T} P^{l} \\
P^{l} A_{1}^{i}+\frac{\varphi \varepsilon_{2}-\varepsilon_{1}}{2} I & P^{l} \tilde{W}_{1}^{i} & P^{l} \bar{W}_{2}^{i} & P^{l}-\varepsilon_{2} I
\end{array}\right] \prec 0
$$


Applying Lemma 3.1 to (97) leads to (89), which completes the proof.

\section{Illustrative examples}

\subsection{Academic example}

Let us consider the TS system provided in the example at the end of Section 3.2, and let us notice that the approach proposed by Chadli and Karimi (2013) cannot be applied to this example, since (15) leads to $E=0$ which, combined with (11) and (14), gives:

$$
T B^{i}-K^{i} F=B^{i}=0
$$

which is false. On the other hand, applying the design procedure described in Section 3.3, the following UIO matrices are obtained with $\mu=4.6615$ :

$$
\begin{gathered}
K^{1}=\left(\begin{array}{cc}
-0.4473 & 0.1226 \\
0.1880 & -0.1324 \\
0.0788 & 0.1570
\end{array}\right) \quad K^{2}=\left(\begin{array}{cc}
-0.5237 & 0.1383 \\
0.2313 & -0.1431 \\
0.0790 & 0.1685
\end{array}\right) \quad K^{3}=\left(\begin{array}{cc}
-0.4872 & 0.1039 \\
0.4589 & -0.1651 \\
0.0063 & 0.1620
\end{array}\right) \\
K^{4}=\left(\begin{array}{cc}
-0.5657 & 0.0992 \\
0.5328 & -0.1693 \\
0.0164 & 0.1855
\end{array}\right) \quad K^{5}=\left(\begin{array}{cc}
-0.5416 & 0.1205 \\
0.1190 & -0.1616 \\
0.3205 & 0.2266
\end{array}\right) \quad K^{6}=\left(\begin{array}{cc}
-0.6522 & 0.1273 \\
0.1654 & -0.1708 \\
0.3576 & 0.2639
\end{array}\right) \\
K^{7}=\left(\begin{array}{cc}
-0.5802 & 0.0860 \\
0.4185 & -0.1661 \\
0.2673 & 0.2233
\end{array}\right) \quad K^{8}=\left(\begin{array}{cc}
-0.6246 & -0.0264 \\
0.5515 & -0.0403 \\
0.3749 & 0.2220
\end{array}\right)
\end{gathered}
$$

In order to show the performance of the proposed approach, a simulation has been performed with $x_{0}=(5,-5,2)^{T}, u_{k}=\sin (k / 20), w_{i} \in[-0.1,0.1], i=1, \ldots, 5$, where each $w_{i}$ is generated as a random sequence, $s \in[0,1]$ as shown in Figure 1 (corresponding to the activation functions $\rho_{i}, i=$ $1, \ldots, 8$, depicted in Figure 2), and $f_{k}$ as follows:

$$
f_{k}=\left\{\begin{array}{cc}
0 & k \leq 20 \\
0.5 & 20<k \leq 40 \\
0.5+0.5 \sin (2 \pi k / 40) & \text { else }
\end{array}\right.
$$

As expected, the results shown in Figures 3 and 4 demonstrate the convergence of the UIO in the estimation of both the state $x_{k}$ and the fault $f_{k}$, thus proving the effectiveness of the developed method.

\subsection{Multi-tank system}

R2-1 R3-4 Let us consider a multi-tank system portrayed in Fig. 5. It consists of three separate tanks placed one above the other and equipped with drain valves and level sensors based on hydraulic pressure measurement (Witczak 2014). Each of them has a different cross-section in order to reflect system nonlinearities. The lower bottom tank is a water reservoir for the system. A variable speed water pump is used to fill the upper tank and the water outflows the tanks due to the gravity. The 


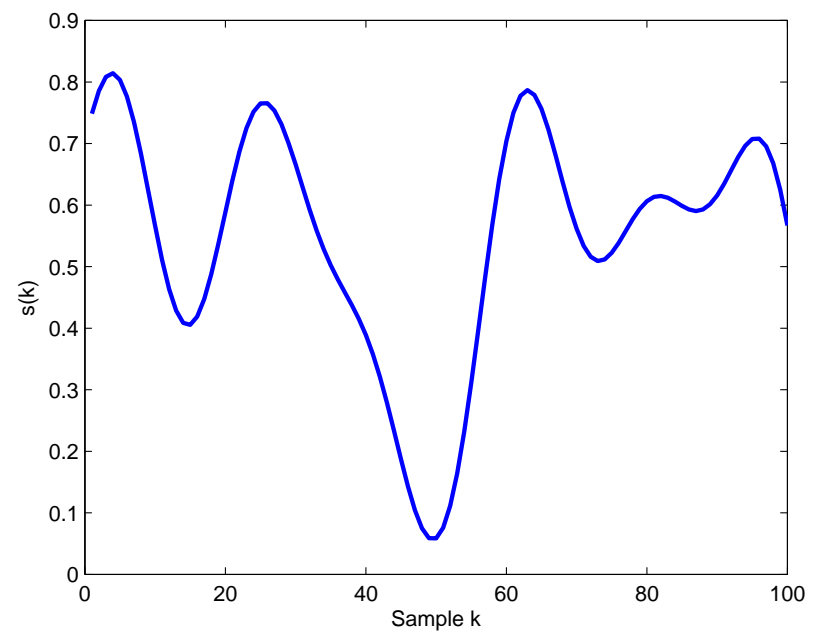

Figure 1. Premise variable $s(k)$.
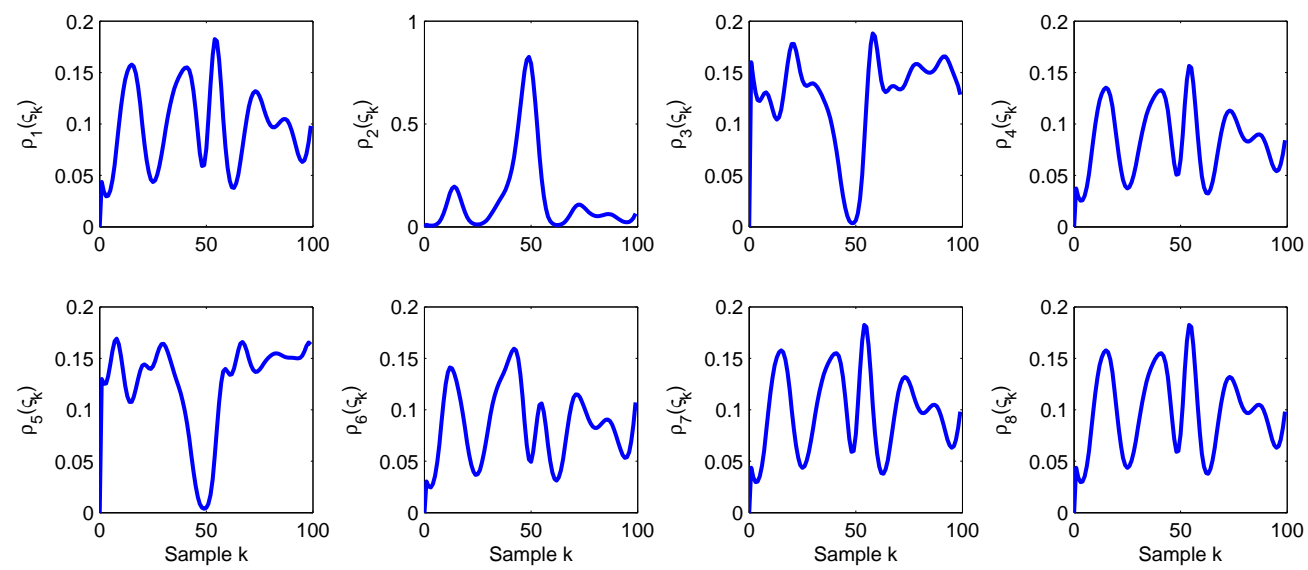

Figure 2. Activation functions $\rho(k)$.

nonlinear discrete-time model of the multi-tank system is given by (Witczak 2014):

$$
\left\{\begin{array}{l}
h_{1}(k+1)=h_{1}(k)-c_{1} h_{1}(k)^{\alpha}+b u(k) \\
h_{2}(k+1)=h_{2}(k)+\left(c_{3}+c_{4} h_{2}(k)\right)^{-1}\left(c_{2} h_{1}(k)^{\alpha}-c_{5} h_{2}(k)^{\alpha}\right) \\
h_{3}(k+1)=h_{3}(k)+\left(c_{7}-\left(c_{8}-h_{3}(k)\right)^{2}\right)^{-0.5}\left(c_{6} h_{2}(k)^{\alpha}-c_{9} h_{3}(k)^{\alpha}\right)
\end{array}\right.
$$

where the real data-based parameters were identified as follows: $b=1.14, \alpha=0.5, c_{1}=1.15 \cdot 10^{-4}$, $c_{2}=1.01 \cdot 10^{-6}, c_{3}=3.5 \cdot 10^{-3}, c_{4}=3.48 \cdot 10^{-2}, c_{5}=1.20 \cdot 10^{-6}, c_{6}=3.42 \cdot 10^{-5}, c_{7}=1.33 \cdot 10^{-1}$, $c_{8}=3.5 \cdot 10^{-1}$ and $c_{9}=2.80 \cdot 10^{-5}$.

By considering actuator faults $f_{k}$ and an exogenous disturbance vector $w_{k}$ entering into the system through the matrix:

$$
W_{1}=\left(\begin{array}{ccc}
0.001 & 0 & 0 \\
0 & 0 & 0 \\
0 & 0 & 0
\end{array}\right)
$$



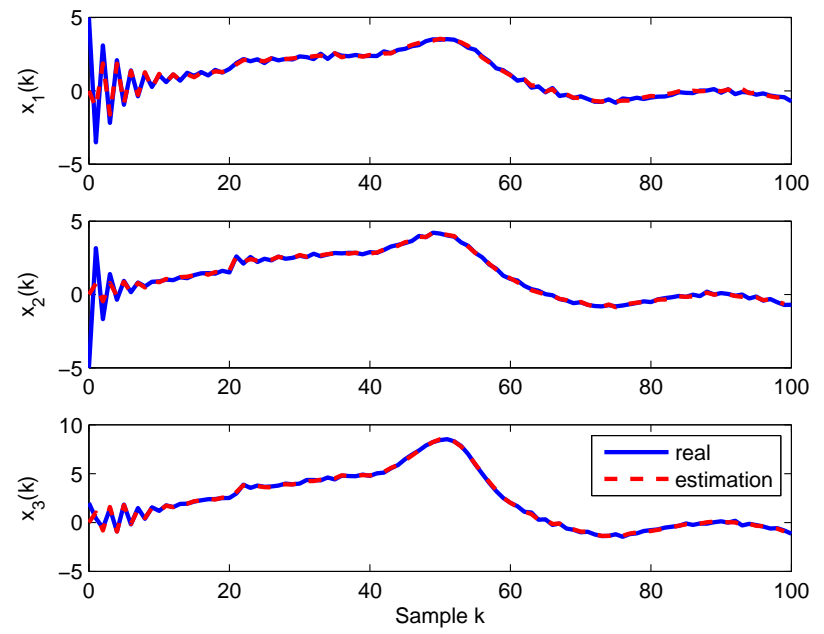

Figure 3. State $x_{k}$ and its estimation $\hat{x}_{k}$ using the proposed UIO.

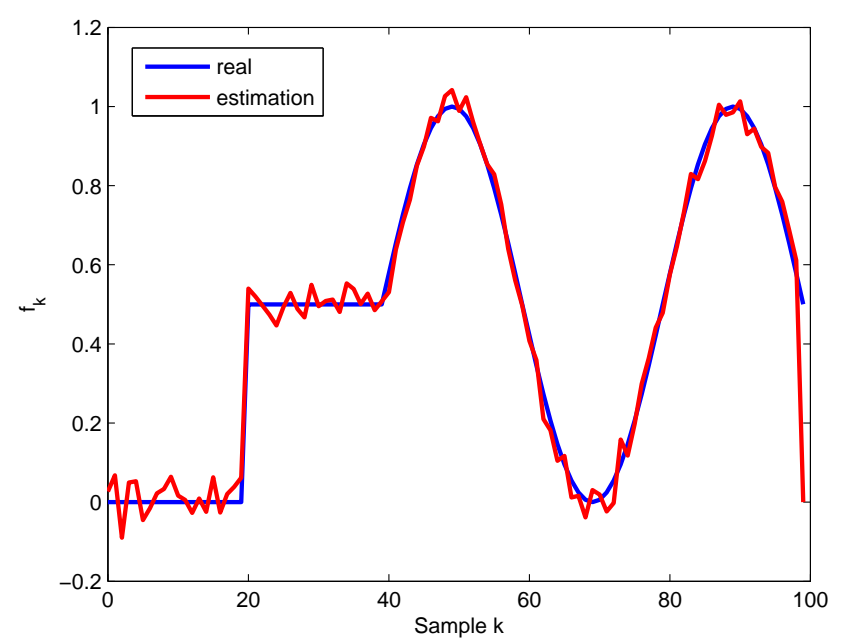

Figure 4. Fault $f_{k}$ and its estimation $\hat{f}_{k}$ using the proposed UIO.

one can rewrite (99) as (1), with $x_{k}=\left[h_{1}(k), h_{2}(k), h_{3}(k)\right]^{T}, u_{k}=u(k)$, and:

$$
A\left(s_{k}\right)=\left(\begin{array}{ccc}
s_{k}^{(1)} & 0 & 0 \\
s_{k}^{(2)} & s_{k}^{(3)} & 0 \\
0 & s_{k}^{(4)} & s_{k}^{(5)}
\end{array}\right) \quad B=\left(\begin{array}{l}
b \\
0 \\
0
\end{array}\right)
$$




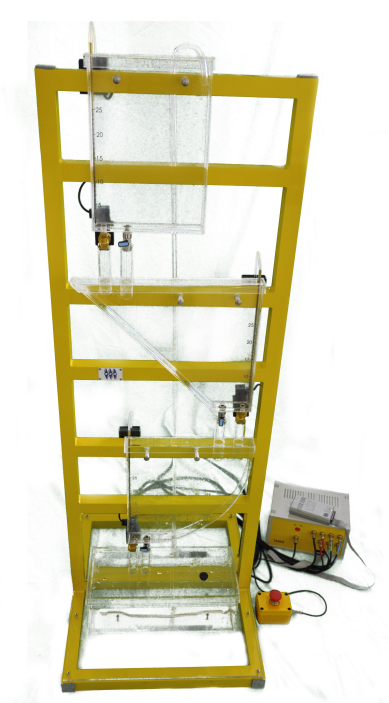

Figure 5. Multi-Tank system.

where:

$$
\begin{aligned}
& s_{k}^{(1)}=1-c_{1} h_{1}(k)^{\alpha-1} \\
& s_{k}^{(2)}=c_{2}\left(c_{3}+c_{4} h_{2}(k)\right)^{-1} h_{1}(k)^{\alpha-1} \\
& s_{k}^{(3)}=1-c_{5}\left(c_{3}+c_{4} h_{2}(k)\right)^{-1} h_{2}(k)^{\alpha-1} \\
& s_{k}^{(4)}=c_{6}\left(c_{7}-\left(c_{8}-h_{3}(k)\right)^{2}\right)^{-0.5} h_{2}(k)^{\alpha-1} \\
& s_{k}^{(5)}=1-c_{9}\left(c_{7}-\left(c_{8}-h_{3}(k)\right)^{2}\right)^{-0.5} h_{3}(k)^{\alpha-1}
\end{aligned}
$$

It is assumed that noisy measurements of $h_{1}(k)$ and $h_{2}(k)$ are available, i.e., the output equation (2) is characterized by the matrices:

$$
C=\left(\begin{array}{lll}
1 & 0 & 0 \\
0 & 1 & 0
\end{array}\right) \quad W_{2}=\left(\begin{array}{ccc}
0 & 0.003 & 0 \\
0 & 0 & 0.003
\end{array}\right)
$$

It is easy to check that (18) is verified, since:

$$
C B=\left(\begin{array}{l}
b \\
0
\end{array}\right)
$$

has rank 1, which allows calculating $H$ using (28):

$$
H=\left(\frac{1}{b}\right.
$$

Hence, it follows from (31)-(34) that:

$$
\bar{A}\left(s_{k-1}\right)=\left(\begin{array}{ccc}
0 & 0 & 0 \\
s_{k-1}^{(2)} & s_{k-1}^{(3)} & 0 \\
0 & s_{k-1}^{(4)} & s_{k-1}^{(5)}
\end{array}\right) \quad \bar{H}=\left(\begin{array}{ll}
1 & 0 \\
0 & 0 \\
0 & 0
\end{array}\right)
$$




$$
\bar{W}_{1}=\left(\begin{array}{ccc}
0 & 0 & 0 \\
0 & 0 & 0 \\
0 & 0 & 0
\end{array}\right) \quad \bar{W}_{2}=\left(\begin{array}{ccc}
0 & 0.003 & 0 \\
0 & 0 & 0 \\
0 & 0 & 0
\end{array}\right)
$$

By defining the new premise variables $\varsigma_{1}=s_{k-1}^{(2)}, \varsigma_{2}=s_{k-1}^{(3)}, \varsigma_{3}=s_{k-1}^{(4)}$ and $\varsigma_{4}=s_{k-1}^{(5)}$ and considering that $h_{i} \in\left[h_{\min }, h_{\max }\right], i=1,2,3$, (35)-(36) becomes a set of $\bar{M}=16$ subsystems, obtained considering all the possible combinations of extreme values of the intervals $\left[\varsigma_{i}^{\min }, \varsigma_{i}^{\max }\right]$, $i=1,2,3,4$, calculated as follows:

$$
\begin{array}{cc}
\varsigma_{1}^{\min }=c_{2}\left(c_{3}+c_{4}+h_{\max }\right)^{-1} h_{\max }^{\alpha-1} & \varsigma_{1}^{\max }=c_{2}\left(c_{3}+c_{4}+h_{\min }\right)^{-1} h_{\min }^{\alpha-1} \\
\varsigma_{2}^{\min }=1-c_{5}\left(c_{3}+c_{4} h_{\min }\right)^{-1} h_{\min }^{\alpha-1} & \varsigma_{2}^{\max }=1-c_{5}\left(c_{3}+c_{4} h_{\max }\right)^{-1} h_{\max }^{\alpha-1} \\
\varsigma_{3}^{\min }=c_{6}\left(c_{7}-\left(c_{8}-h_{\max }\right)^{2}\right)^{-0.5} h_{\max }^{\alpha-1} & \varsigma_{3}^{\max }=c_{6}\left(c_{7}-\left(c_{8}-h_{\min }\right)^{2}\right)^{-0.5} h_{\min }^{\alpha-1} \\
\varsigma_{4}^{\min }=1-c_{9}\left(c_{7}-\left(c_{8}-h_{\min }\right)^{2}\right)^{-0.5} h_{\min }^{\alpha-1} & \varsigma_{4}^{\max }=1-c_{9}\left(c_{7}-\left(c_{8}-h_{\max }\right)^{2}\right)^{-0.5} h_{\max }^{\alpha-1}
\end{array}
$$

Due to the noise and the unavailability of measurements for $h_{3}(k)$, the case of unmeasurable premise variables should be considered, as described in Section 3.6. In this case, (86) reads as follows:

$$
\Delta(\cdot)=\left(\begin{array}{c}
0 \\
\left(s_{k}^{(2)}-\hat{s}_{k}^{(2)}\right) \\
\left(s_{k}^{(4)}-\hat{s}_{k}^{(4)}\right) \\
h_{1}(k)+\left(\hat{s}_{k}^{(3)}-s_{k}^{(3)}\right) \\
h_{2}(k)+\left(\hat{s}_{k}^{(5)}-s_{k}^{(5)}\right) \\
h_{2}(k) \\
h_{3}(k)
\end{array}\right)
$$

The properties of $\Delta(\cdot)$ being one-sided Lipschitz and quadratic inner-bounded, i.e. (87)-(88), have been verified with $\rho=0.0012, \sigma=10^{-11}$ and $\varphi=0.2396$.

Applying the design procedure described in Section 3.6, a feasible solution has been obtained with $\tau=5, \mu=1.35 \cdot 10^{-3}, \varepsilon_{1}=6.3829$ and $\varepsilon_{2}=26.89$.

All of the experiments have been performed with the real system using the following parameters: $x_{0}=[0.001,0.001,0.001]^{T}, \hat{x}_{0}=[0.05,0.05,0.01]^{T}, u_{k}=9 \cdot 10^{-5}$, and $f_{k}$ defined as:

$$
f_{k}=\left\{\begin{array}{cc}
-0.33 \cdot u_{k} & 10001<k \leq 15000 \\
0 & \text { otherwise }
\end{array}\right.
$$

Fig. 6 shows the activation functions $\rho(k)$ of the described system. Figs. 7-9 present the states and their estimates. Considering that the water level in the third tank is unmeasurable and that it varies in the interval $[0,0.35(\mathrm{~m})]$ the results are satisfactory, as it can be clearly observed in Fig. 10 depicting the state estimation error. Indeed, the estimation error does not exceed $1 \mathrm{~cm}$ which, taking into account the permanent water flow as well as the sensor measurement imprecision, should be perceived as a good result. Fig. 11 shows the actuator loss of effectiveness fault $f_{k}$ and its robust estimate $\hat{f}_{k}$ (red dashed line). The obtained results have been compared with the ones obtained using a linear UIO, as proposed by Witczak (2014). In this case, the matrices describing the linear model have been taken from the documentation (INTECO 2013). The fault estimation obtained using the linear UIO is shown in Fig. 11 (black dashed line). From these results, it is clear that the proposed UIO performs significantly better than the linear one. Indeed, Fig. 12 clearly shows that the fault estimation error associated with the proposed approach is relatively small. 

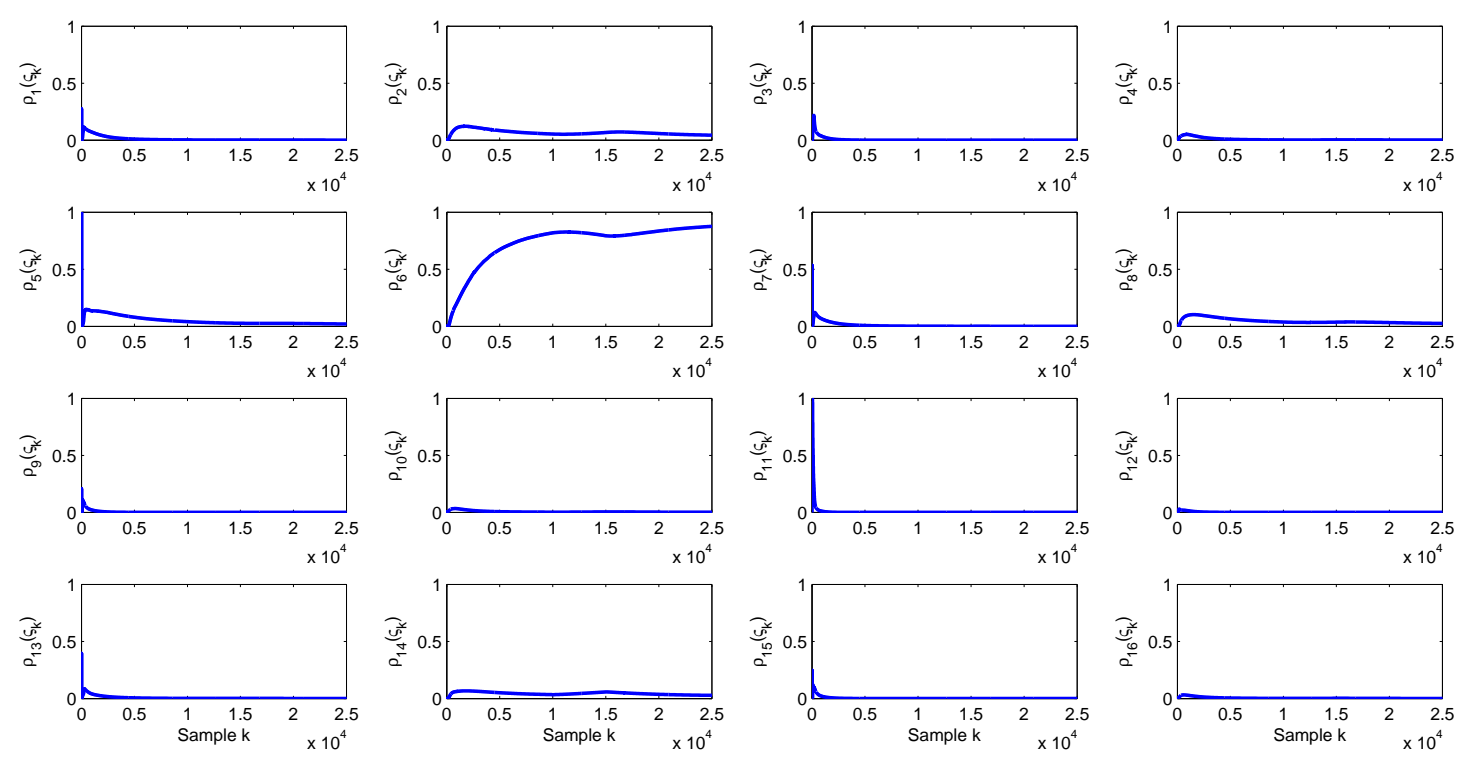

Figure 6. Activation functions $\rho(k)$.

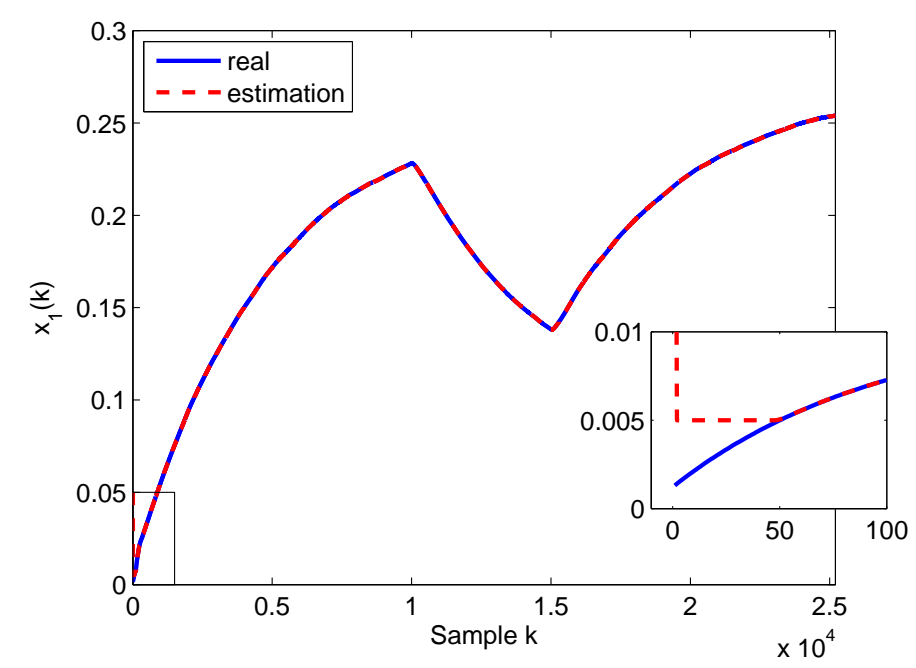

Figure 7. State $x_{1, k}$ and its estimate using the proposed UIO - first tank.

\section{Conclusions}

In this paper, an UIO for TS systems has been proposed, with the goal of jointly estimating the state and the unknown actuator faults. Differently from recent results appeared in the literature, the proposed approach can deal with TS systems whose output equation matrices are not constant. Moreover, instead of eliminating the external disturbances, their influence on the UIO performance is minimized, with the advantage of not requiring the perfect knowledge of the disturbance distribution matrices. The whole design procedure, that aims at achieving convergence of the estimation error either with any rate, or with a guaranteed decay rate, boils down to solve a set of LMIs, a problem that can be efficiently solved using the solvers available nowadays. The case of activation functions which depend on unmeasurable premise variables has been also considered. The effectiveness of the proposed method has been demonstrated using an academical example and a real system application. In particular, a multi-tank system was employed to perform a comparison of the proposed TS UIO with a linear one. The obtained results clearly show that the proposed approach performs significantly better than the linear one. 


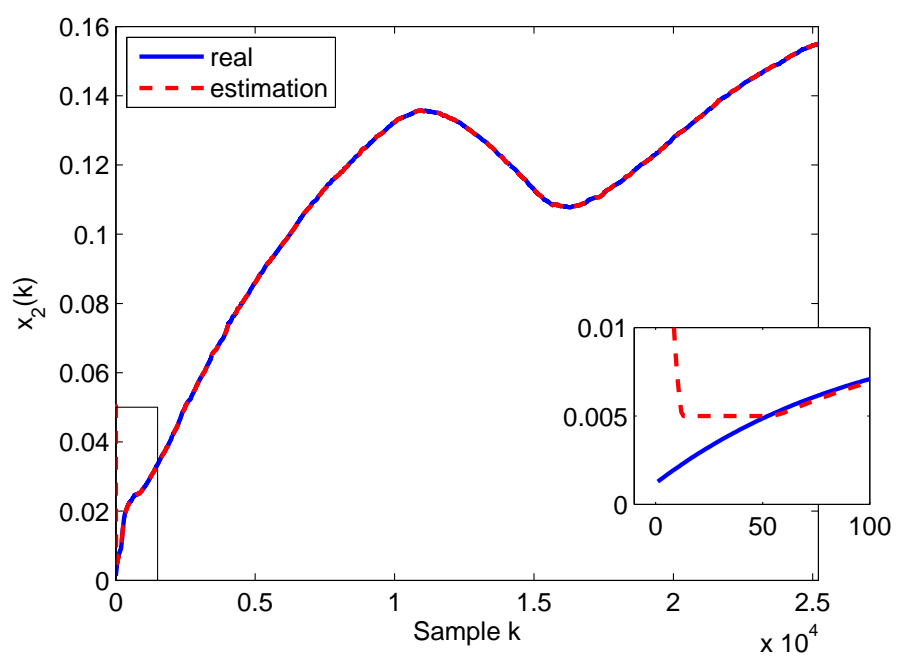

Figure 8. State $x_{2, k}$ and its estimate using the proposed UIO - second tank.

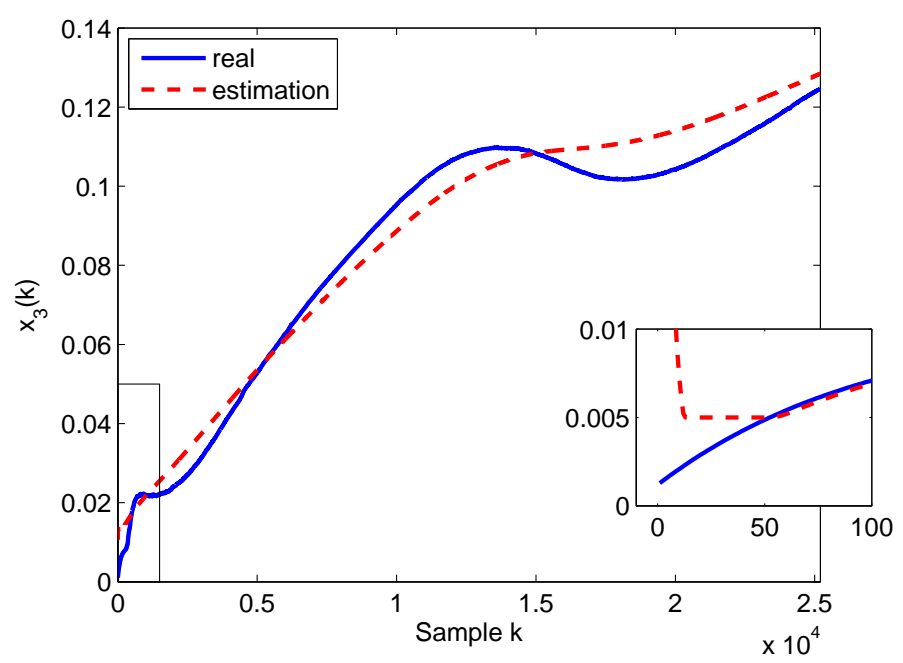

Figure 9. State $x_{3, k}$ and its estimate using the proposed UIO - third tank.

\section{Acknowledgments}

This work has been funded by the National Science Centre in Poland under the grant 2013/11/B/ST7/01110, by the Spanish Government (MINECO) through the project CICYT ECOCIS (ref. DPI2013-48243-C2-1-R), by MINECO and FEDER through the project CICYT HARCRICS (ref. DPI2014-58104-R), by AGAUR through the contracts FI-DGR 2014 (ref. 2014FI_B1 00172) and FIDGR 2015 (ref. 2015FI_B2 00171), and by the DGR of Generalitat de Catalunya (SAC group Ref. 2014/SGR/374).

\section{References}

Abbaszadeh, M., and Marquez, H.J. (2009), "LMI optimization approach to robust $\mathcal{H}_{\infty}$ observer design and static output feedback stabilization for discrete-time nonlinear uncertain systems," International Journal of Robust and Nonlinear Control, 19(3), 313-340.

Bouattour, M., Chadli, M., El Hajjaji, A., and Chaabane, M. (2010), "Estimation of state, actuator and sensor faults for TS models," in Proceedings of the 49th IEEE Conference on Decision and Control (CDC), pp. 1613-1618.

Brahim, A., Dhahri, S., Hmida, F., and Sellami, A. (2015), "An $H_{\infty}$ sliding mode observer for Takagi-Sugeno 


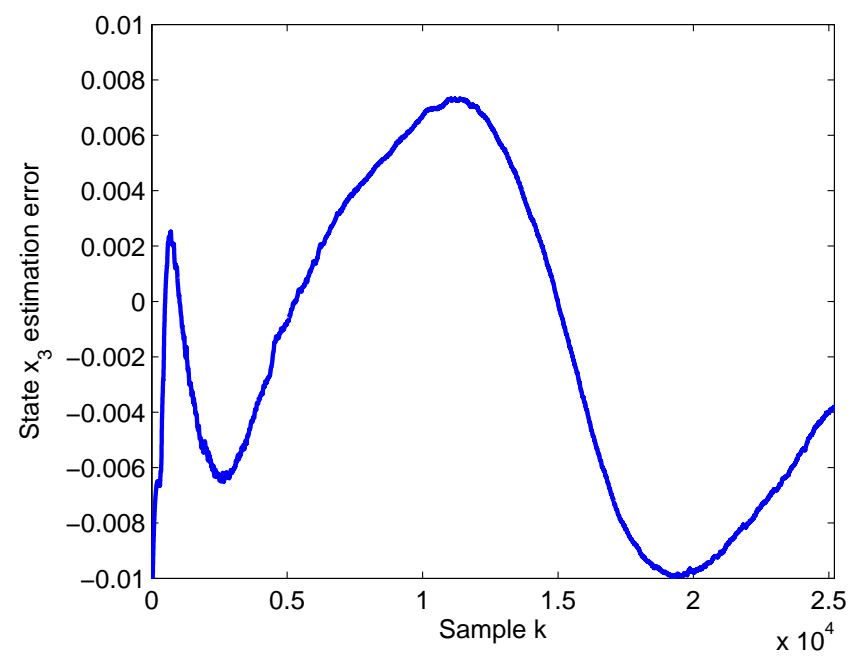

Figure 10. State estimation error using the proposed UIO - third tank.

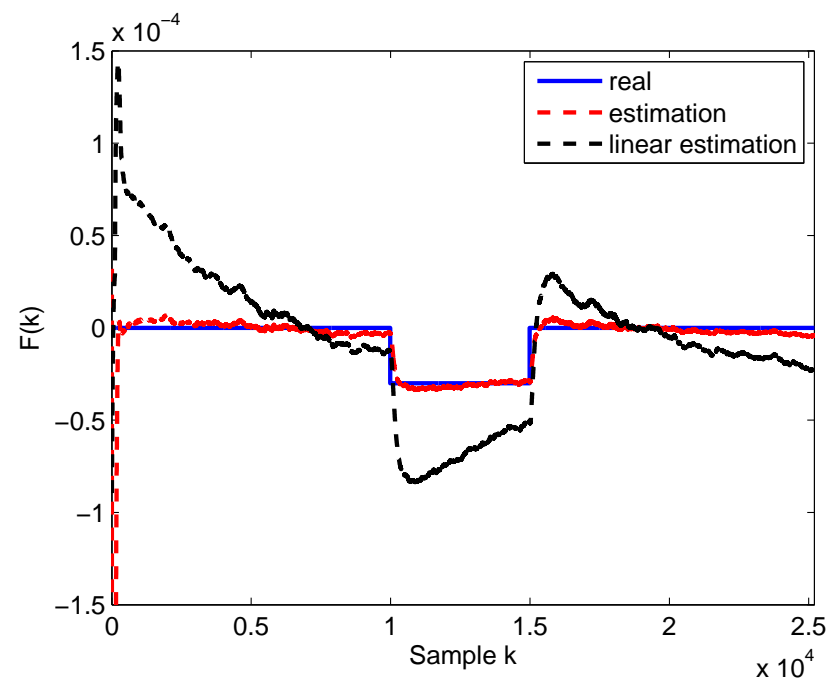

Figure 11. Fault $f_{k}$ and its estimation $\hat{f}_{k}$ using the proposed and linear UIO.

nonlinear systems with simultaneous actuator and sensor faults," International Journal of Applied Mathematics and Computer Science, 25(3), 547-559.

Chadli, M. (2010), "An LMI approach to design observer for unknown inputs Takagi-Sugeno fuzzy models," Asian Journal of Control, 12(4), 524-530.

Chadli, M., Akhenak, A., Ragot, J., and Maquin, D. (2009), "State and unknown input estimation for discrete time multiple model," Journal of the Franklin Institute, 346(6), 593-610.

Chadli, M., and Karimi, H.R. (2013), "Robust observer design for unknown inputs Takagi-Sugeno models," IEEE Transactions on Fuzzy Systems, 21(1), 158-164.

Chen, W., and Saif, M. (2007), "Design of a TS based fuzzy nonlinear unknown input observer with fault diagnosis application," in Proceedings of the 24th American Control Conference, pp. 2545-2550.

Chen, W., and Saif, M. (2010), "Fuzzy nonlinear unknown input observer design with fault diagnosis applications," Journal of Vibration and Control, 16(3), 377-401.

Cotton, N.J., and Wilamowski, B.M. (2010), "Compensation of sensors nonlinearity with neural networks," in Proceedings of the 24th IEEE International Conference on Advanced Information Networking and Applications, pp. 1210-1217.

de Oliveira, M.C., Bernussou, J., and Geromel, J.C. (1999), “A new discrete-time robust stability condition,” Systems and Control Letters, 37(4), 261-265.

Ducard, G., Fault-tolerant flight control and guidance systems: practical methods for small unmanned aerial vehi- 


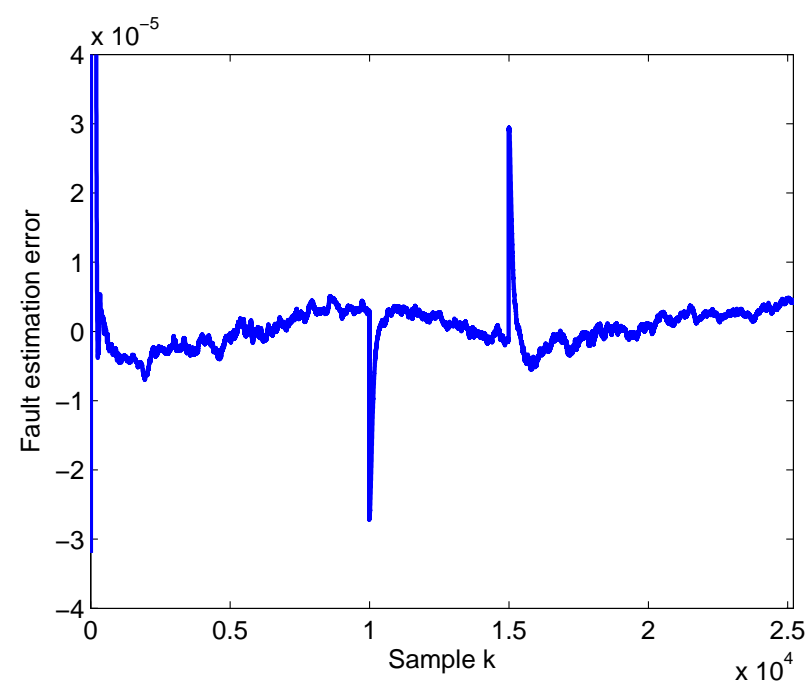

Figure 12. Fault estimation error using the proposed UIO.

cles, Berlin: Springer-Verlag (2009).

Elsner, L., Monov, V., and Szulc, T. (2002), "On some properties of convex matrix sets characterized by P-matrices and block P-matrices," Linear and Multilinear Algebra, 50, 199-218.

Elsner, L., and Szulc, T. (1998), "Convex combinations of matrices - nonsingularity and Schur stability characterizations," Linear and Multilinear Algebra, 44, 301-312.

Elsner, L., and Szulc, T. (2002), "Convex sets of Schur stable and stable matrices," Linear and Multilinear Algebra, $48,1-19$.

Feng, G. (2006), "A survey on analysis and design of model-based fuzzy control systems," IEEE Transactions on Fuzzy Systems, 14(5), 676-697.

Fonod, R., Henry, D., Charbonnel, C., and Bornschlegl, E. (2014), “A class of nonlinear unknown input observer for fault diagnosis: application to fault tolerant control of an autonomous spacecraft," in Proceedings of the 10th UKACC International Conference on Control, pp. 19-24.

Gillijns, S., and Moor, B.D. (2007), "Unbiased minimum-variance input and state estimation for linear discretetime systems with direct feedthrough," Automatica, 43(5), 934-937.

Guerra, T.M., Kruszewski, A., and Lauber, J. (2009), "Discrete Takagi-Sugeno models for control: where are we?," Annual Reviews in Control, 33(1), 37-47.

Hwang, I., Kim, S., Kim, Y., and Seah, C.E. (2010), "A survey of fault detection, isolation, and reconfiguration methods," IEEE Transactions on Control Systems Technology, 18(3), 636-653.

Ichalal, D., Marx, B., Ragot, J., and Maquin, D. (2009), "An approach for the state estimation of Takagi-Sugeno models and application to sensor fault diagnosis," in Proceedings of the 48th IEEE Conference on Decision and Control (CDC), pp. 7789-7794.

Ichalal, D., Marx, B., Ragot, J., and Maquin, D. (2010), "State estimation of Takagi-Sugeno systems with unmeasurable premise variables," IET Control Theory and Applications, 4(5), 897-908.

INTECO,, Multitank System - User's manual, www.inteco.com.pl (2013).

Jia, Q.X., Zhang, Y.C., Guan, Y., and Wu, L.N. (2011), "Robust nonlinear unknown input observer-based fault diagnosis for satellite attitude control system," in Proceedings of the 10th UKACC International Conference on Control, pp. 345-350.

Johansen, T.A., Shorten, R., and Murray-Smith, R. (2000), "On the interpretation and identification of dynamic Takagi-Sugeno models," IEEE Transactions on Fuzzy Systems, 8(3), 297-313.

Johnson, C.R., and Tsatsomeros, M.J. (1995), "Convex sets of nonsingular and P-matrices," Linear and Multilinear Algebra, 38, 233-239.

K. Zhang, B.J., and Shi, P. (2009), "A new approach to observer-based fault-tolerant controller design for TakagiSugeno fuzzy systems with state delay," Circuits, Systems and Signal Processing, 28(5), 679-697.

Keller, J.Y., and Darouach, M. (1999), "Two-stage Kalman estimator with unknown exogenous inputs," Automatica, 35(2), 339-342.

Kolodziejczak, B., and Szulc, T. (1999), "Convex combinations of matrices - Full rank characterization," Linear Algebra and its Applications, 287(1-3), 215-222. 
Li, H., and Fu, M. (1997), “A linear matrix inequality approach to robust $\mathcal{H}_{\infty}$ filtering," IEEE Transactions on Signal Processing, 45(9), 2338-2350.

Mahmoud, M., Jiang, J., and Zhang, Y., Active fault tolerant control systems, Berlin: Springer-Verlag (2003).

Moodi, H., and Farrokhi, M. (2013), "Robust observer design for Sugeno systems with incremental quadratic nonlinearity in the consequent," International Journal of Applied Mathematics and Computer Science, 23(4), 711-723.

Noura, H., Theilliol, D., Ponsart, J.C., and Chamseddine, A., Fault-tolerant control systems: design and practical applications, Berlin: Springer-Verlag (2009).

Patan, K., Witczak, M., and Korbicz, J. (2008), "Towards robustness in neural network based fault diagnosis," International Journal of Applied Mathematics and Computer Science, 18(4), 443-454.

Puig, V. (2010), "Fault diagnosis and fault tolerant control using set-membership approaches: application to real case studies," International Journal of Applied Mathematics and Computer Science, 20(4), 619-635.

Rotondo, D., Puig, V., Nejjari, F., and Witczak, M. (2015), “Automated generation and comparison of TakagiSugeno and polytopic quasi-LPV models," Fuzzy Sets and Systems, 277, 44-64.

Rotondo, D., Reppa, V., Puig, V., and Nejjari, F. (2014), “Adaptive observer for switching linear parameter-varying (LPV) systems," in Preprints of the 19th IFAC World Congress, pp. 1471-1476.

Samy, I., Postlethwaite, I., and Gu, D.W. (2011), "Survey and application of sensor fault detection and isolation schemes," Control Engineering Practice, 19(7), 658-674.

Simani, S., Fantuzzi, C., and Patton, R., Model-based fault diagnosis in dynamic systems, Springer, London (2003).

Takagi, T., and Sugeno, M. (1985), "Fuzzy identification of systems and its application to modeling and control," IEEE Transactions on Systems, Man, and Cybernetics, SMC-15, 116-132.

Tanaka, K., and Wang, H.O., Fuzzy control systems design and analysis: a LMI approach, New York: Wiley (2001).

Tuan, H.D., Apkarian, P., Narikiyo, T., and Yamamoto, Y. (2001), "Parameterized linear matrix inequality techniques in fuzzy control system design," IEEE Transactions on Fuzzy Systems, 9(2), 324-332.

Vizer, D., Mercere, G., Prot, O., and Ramos, J. (2013), “A local approach for black-box and gray-box LPV system identification," in Proceedings of the 12th European Control Conference, pp. 1916-1921.

Wang, H.O., Tanaka, K., and Griffin, M.F. (1996), "An approach to fuzzy control of nonlinear systems: stability and design issues," IEEE Transactions on Fuzzy Systems, 4(1), 14-23.

Wang, S.H., Wang, E., and Dorato, P. (1975), "Observing the states of systems with unmeasurable disturbances," IEEE Transactions on Automatic Control, 20(5), 716-717.

Witczak, M., Modelling and estimation strategies for fault diagnosis of non-linear systems: from analytical to soft computing approaches, Berlin: Springer-Verlag (2007).

Witczak, M., Fault diagnosis and fault-tolerant control strategies for non-linear systems, Springer (2014).

Witczak, M., Buciakowski, M., Puig, V., Rotondo, D., and Nejjari, F. (2015), "An LMI approach to robust fault estimation for a class of nonlinear systems," International Journal of Robust and Nonlinear Control.

Witczak, M., Rotondo, D., Puig, V., and Witczak, P. (2015), "A practical test for assessing the reachability of discrete-time Takagi-Sugeno fuzzy systems," Journal of the Franklin Institute, 352(12), 5936-5951.

$\mathrm{Xu}$, D., Jiang, B., and Shi, P. (2012), "Nonlinear actuator fault estimation observer: an inverse system approach via T-S fuzzy model," International Journal of Applied Mathematics and Computer Science, 22(1), 183-196.

Zemouche, A., Boutayeb, M., and Iulia Bara, G. (2008), "Observers for a class of Lipschitz systems with extension to $\mathcal{H}_{\infty}$ performance analysis," Systems and Control Letters, 57(1), 18-27.

Zhang, W., Su, H., Zhu, F., and Yue, D. (2012), "A note on observers for discrete-time Lipschitz nonlinear systems," IEEE Transactions on circuits and systems - II: Express Briefs, 59(2), 123-127.

Zhang, X., Polycarpou, M.M., and Parisini, T. (2010), "Fault diagnosis of a class of nonlinear uncertain systems with Lipschitz nonlinearities using adaptive estimation," Automatica, 46(2), 290-299.

Zhang, Y., and Jiang, J. (2008), "Bibliographical review on reconfigurable fault-tolerant control systems," Annual Reviews in Control, 32(2), 229-252. 